This is an accepted manuscript of an article published by Taylor \& Francis

in Communications in Statistics - Theory and Methods (Volume 43, Issue 21, 2014, pp. 4589-4611).

Published version available at: http://dx.doi.org/10.1080/03610926.2012.737495

\title{
A parametric test of perfect ranking in balanced ranked set sampling
}

\author{
Ehsan Zamanzade ${ }^{\mathrm{a}, 1}$, Nasser Reza Arghami ${ }^{\mathrm{b}}$ and Michael Vock ${ }^{\mathrm{c}}$ \\ ${ }^{a}$ Department of Statistics, University of Isfahan, Isfahan 81744, Iran. \\ ${ }^{\mathrm{b}}$ Department of Statistics, Faculty of Mathematical Sciences, Ferdowsi University of Mashhad, Mashhad, \\ Iran. \\ ${ }^{c}$ Institute of Mathematical Statistics and Actuarial Science, University of Bern, Sidlerstrasse 5, CH-3012 \\ Bern, Switzerland.
}

\begin{abstract}
:
Many techniques based on data which are drawn by Ranked Set Sampling (RSS) scheme assume that the ranking of observations is perfect. Therefore it is essential to develop some methods for testing this assumption. In this paper, we propose a parametric location-scale free test for assessing the assumption of perfect ranking. The results of a simulation study in two special cases of normal and exponential distributions indicate that the proposed test performs well in comparison with its leading competitors.
\end{abstract}

Keywords: Ranked set sampling, Test, assumption of perfect ranking

\section{Introduction}

When actual measuring of observations is expensive or time-consuming but ranking them (without actual measurement, e.g. by visual inspection) is cheap and easy, ranked set sampling (RSS), even in small groups, provides an efficient sampling method in comparison with simple random sampling (SRS). The notion of ranked set sampling was suggested, for the first time, by McIntyre (1952) for estimation of average harvest, when he realized that it is easy to rank amounts of harvest in different farms while it is hard to actually measure them. Ranked set sampling can be balanced or unbalanced. In the balanced case (BRSS), one draws $k$ samples of size $k$, orders each sample in increasing order and then selects the $i^{\text {th }}$ smallest unit from the $i^{\text {th }}$ sample for measurement. This procedure is repeated $n$ times (cycles) to produce a balanced ranked set sample of size $n k$. Unbalanced ranked set sampling (UBRSS) differs from BRSS in that the different order statistics are not necessarily selected the same number of times.

There has been a lot of research on RSS in both nonparametric and parametric cases since McIntyre (1952) introduced it. In the nonparametric case, Takahasi and Wakimoto (1968) proved that estimation of the population mean in RSS is much more efficient than in SRS. Stokes (1980) proposed an estimate for the population variance and proved its efficiency and then Perron and Sinha (2002) improved it in the multi-cycle case. In the parametric case, Sengupta and Mukhuti (2006) proposed an improved estimator of variance based on BRSS for the exponential population; Bhoj and Asanullah

\footnotetext{
${ }^{1}$ Corresponding author.

Email address: E.Zamanzade@sci.ui.ac.ir(E. Zamanzade), Arghami_nr@yahoo.com (N.R Arghami) and Michael.vock@stat.unibe.ch (M. Vock)
} 
(1996) suggested BLUE based on RSS for generalized geometric distribution and then Balakrishnan and Li (2005) improved it by means of Ordered Ranked Set Sampling (ORSS). A short but useful description of works on RSS can be found in Wolfe (2004).

Perfect ranking is one of the fundamental assumptions of RSS, under which the units in each sample are ranked without error. Generally, the procedures based on RSS remain valid if this assumption is slightly violated. Many authors have tried to show that their proposed techniques based on RSS remain valid or at least as effective as those based on SRS, when the assumption of perfect ranking is moderately relaxed. Obviously, if this assumption is violated, the efficiency and even validity of those techniques cannot be guaranteed. Therefore, in this situation, in order to have valid inference, some robust techniques should be used in practice. Frey et al (2007) gives two nice examples in both nonparametric and parametric cases, in which considerable judgment ranking error causes changes in inference methods. Based on the above argument, existence of some test for assessing perfect ranking seems to be vital. Surprisingly, it is only recently that we see that some tests for perfect ranking have been introduced. Frey et al (2007) and Li and Balakrishnan (2008) independently proposed some nonparametric (distribution-free) tests for perfect ranking. Vock and Balakrishnan (2011) improved one of the Li and Balakrishnan (2008) tests and made some comparisons among these tests, using Monte Carlo simulations. Furthermore, Vock and Balakrishnan (2012) generalized another test proposed by $\mathrm{Li}$ and Balakrishnan (2008) and showed that their generalized test is equivalent to the test proposed by Frey et al (2007).

We organize the rest of the paper as follows: In Section 2 we introduce our proposed test and prove a theorem for easy computation of the test statistic. In Section 3, we compare empirical power of the proposed test with nonparametric tests under the assumption of normality and exponentiality. Final remarks and recommendations are provided in section 4 .

We use the following notations in the rest of the paper: Let $X_{i j l}$, $i, j \in\{1, \cdots, k\}, l \in\{1, \cdots, n\}$, be the real value of the $i^{\text {th }}$ observation in the $j^{\text {th }}$ sample (of size $k$ ) of the $l^{\text {th }}$ cycle. $X_{[i] j l}, i, j \in\{1, \cdots, k\}, l \in\{1, \cdots, n\}$, denotes the $i^{\text {th }}$ judgment based ranked observation in the $j^{\text {th }}$ sample (of size $k$ ) of the $l^{\text {th }}$ cycle, as opposed to $X_{(i) j l}$, $i, j \in\{1, \cdots, k\}, l \in\{1, \cdots, n\}$, which denotes the actual $i^{\text {th }}$ order statistic of the $j^{\text {th }}$ sample in the $l^{\text {th }}$ cycle. We should emphasize that $X_{[i] i l}, i \in\{1, \cdots, k\}, l \in\{1, \cdots, n\}$, are the actual measurements.

\section{Introduction of the test statistic}

One of the tests proposed by $\mathrm{Li}$ and Balakrishnan (2008) can be rewritten as:

$$
N_{k, n}=\sum_{l=1}^{n} \sum_{i=1}^{k-1} \sum_{j=i+1}^{k} I\left(X_{[i] i l}>X_{[j] j l}\right) \text {, }
$$

where $I($.$) is the indicator function.$

The above test statistic can be interpreted as follows: If the assumption of perfect ranking is completely satisfied, then $P\left(X_{[i] i l}>X_{[j] j l}\right)<.5$, for all $j>i$, thus it is 
expected that the above test statistic gets moderately small if the assumption of perfect ranking is satisfied. Therefore large values of $N_{k, n}$ may be regarded as a symptom of imperfect ranking and thus the hypothesis of perfect ranking is rejected for large enough values of $N_{k, n}$. Vock and Balakrishnan (2011) noticed that the test statistic in (1) just compares actual measurements $\left(X_{[i] j l}, X_{[j] j l}\right)$ that are in the same cycle. Thus they improved $N_{k, n}$ by comparing $X_{[i] i l}$ with $X_{[j] j h}$ for $j>i$ and $l, h \in\{1,2,3, \cdots, n\}$. Their proposed test statistic thus becomes

$J_{k, n}=\sum_{h=1}^{n} \sum_{l=1}^{n} \sum_{i=1}^{k-1} \sum_{j=i+1}^{k} I\left(X_{[i] l i}>X_{[j] j h}\right)$.

Their simulation results proved that this modification improved the power of the test based on $N_{k, n}$.

The only influential factor in the above test statistic is $I\left(X_{[i] i l}>X_{[j] j h}\right)$, which means that it is just important to check if $X_{[i] i l}$ is greater than $X_{[j] j l}$ or not and that the difference between $X_{[i] i l}, X_{[j] j h}$ is ignored. Obviously, a large difference $X_{[1] 11}-X_{[2] 21}$ is more indicative of imperfect ranking, than a small difference. Thus in order to take the difference between $X_{[i] i l}$ and $X_{[j] j h}$ into account, the following test statistic is proposed:

$$
D_{k, n}=\sum_{h=1}^{n} \sum_{l=1}^{n} \sum_{i=1}^{k-1} \sum_{j=i+1}^{k} \frac{\left(X_{[i j i l}-X_{[j] j h}\right) I\left(X_{[i] i l}>X_{[j] j h}\right)}{E\left(\left(X_{[i] l i}-X_{[j] j h}\right) I\left(X_{[i] i l}>X_{[j] j h}\right)\right)}
$$

where $E($.$) is the expected value operator under perfect ranking.$

Large values of $D_{k, n}$ can be regarded as a symptom of imperfect ranking, thus the hypothesis of perfect ranking should be rejected for large enough values of $D_{k, n}$.

If the distribution of the population under study belongs to a location-scale family, then the above test statistic may be rewritten as:

$$
D_{k, n}=\sum_{h=1}^{n} \sum_{l=1}^{n} \sum_{i=1}^{k-1} \sum_{j=i+1}^{k} \frac{\left(X_{[i] i l}-X_{[j] j h}\right) I\left(X_{[i] j l}>X_{[j] j h}\right)}{E_{\mu=0, \sigma=1}\left(\left(X_{[i] i l}-X_{[j] j h}\right) I\left(X_{[i] i l}>X_{[j] j h}\right)\right) \sigma}
$$

where $\mu, \sigma$ are location and scale parameters, respectively.

Clearly, the test statistic in (4) is location-free and it will be scale-free, if an equivariant estimator is used for the estimation of $\sigma$.

Remark 1: It is conceivable that under some circumstances the pattern and the probability of error in judgment ranking may depend on the unknown parameters. That of course does not invalidate the test since the critical values are obtained under the assumption of perfect ranking. The power of the test, in such circumstances, may depend on the unknown parameters. 
Remark 2: It should be noted that the above test statistic in (4) can be generalized to the case of unbalanced ranked set sampling (UBRSS) as: $D_{k, n_{1}, \cdots, n_{k}}=\sum_{i=1}^{k-1} \sum_{j=i+1}^{k} \sum_{l=1}^{n_{i}} \sum_{h=1}^{n_{j}} \frac{\left(X_{[i] l}-X_{[j] h}\right) I\left(X_{[i] l}>X_{[j] h}\right)}{E_{\mu=0, \sigma=1}\left(\left(X_{[i]]}-X_{[j] h}\right) I\left(X_{[i] l}>X_{[j] h}\right)\right) \sigma}$, where $X_{[i] l}$ is the lth of $n_{i}$ observations having judgment-order rank $i$.

Although theoretically it is possible to calculate the test statistic in (4), it is relatively hard. The following theorem helps us to simplify (4).

Theorem 1: Suppose $X_{(1), k}, X_{(2), k}, \cdots, X_{(k), k}$ are independent order statistics from an absolutely continuous distribution and sample sizes of $k$. Then $E\left(\left(X_{(j), k}-X_{(l), k}\right) I\left(X_{(j), k}>X_{(l), k}\right)\right)=$ $\left(\sum_{k_{1}=l}^{k} \frac{j\left(\begin{array}{l}k \\ k_{1}\end{array}\right)\left(\begin{array}{l}k \\ j\end{array}\right)}{\left(k_{1}+j\right)\left(\begin{array}{l}2 k \\ k_{1}+j\end{array}\right)} E_{k_{1}+j, 2 k}+\sum_{k_{1}=j}^{k} \frac{l\left(\begin{array}{l}k \\ k_{1}\end{array}\right)\left(\begin{array}{l}k \\ l\end{array}\right)}{\left(k_{1}+l\right)\left(\begin{array}{l}2 k \\ k_{1}+l\end{array}\right)} E_{k_{1}+l, 2 k}-E_{l, k}\right)$

where $E_{i, k}$ is the expectation of the $i^{\text {th }}$ order statistic in a sample of size $k$.

The proof is provided in the Appendix.

By the above theorem, assuming perfect ranking and that the population distribution belongs to a location-scale family, then the test statistic in (4) simplifies to:

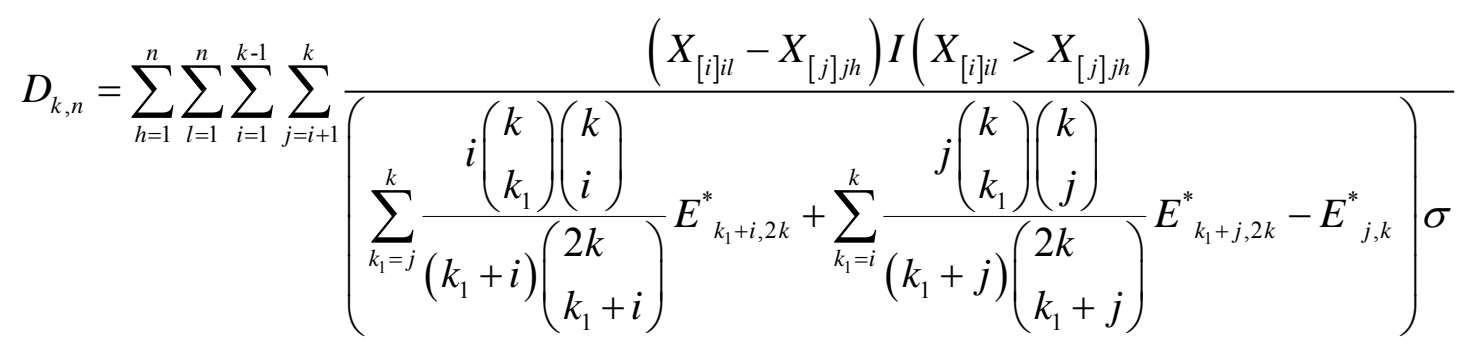

Where $E_{i, k}^{*}$ is expectation of the $i^{\text {th }}$ order statistic in a sample of size $k$ when $\mu=0$ and $\sigma=1$.

\section{Empirical power comparisons}

In this section, we compare our proposed test with its leading competitors, namely:

- The test based on $N_{k, n}$ in (1). 
- The test based on $S_{k, n}$ from Li and Balakrishnan (2008), which has the form of $S_{k, n}=\sum_{l=1}^{n} \sum_{j=1}^{k}\left(i_{j l}-j\right)^{2}$ and rejects the hypothesis of perfect ranking for large values of $S_{k, n}$, where $i_{j l}$ is the judgment order rank used to obtain the $j^{\text {th }}$ order statistic within the $I^{\text {th }}$ cycle.

- The test based on $A_{k, n}$ from Li and Balakrishnan (2008), which has the form of $A_{k, n}=\sum_{l=1}^{n} \sum_{j=1}^{k}\left|i_{j l}-j\right|$ and rejects the hypothesis of perfect ranking for large values of $A_{k, n}$.

- The test based on $W^{*}$ from Frey (2007) with the test statistic $W^{*}=\sum_{j=1}^{k} \sum_{i=1}^{n} j R_{[j] i}$, which rejects the hypothesis of perfect ranking for small values of $W^{*}$, where $R_{[j] i}$ is the rank of $X_{[j] i}$ among all measured values.

- The test based on $J_{k, n}$ in (2).

The powers of the competing tests were directly reported from Vock and Balakrishnan (2011), Tables 3-6, while new simulations were carried out for the power of the test based on $D_{k, n}$.

Because of the undeniable importance of normal and exponential distributions in statistical inference, we consider these two special cases in our study.

\subsection{Empirical power study under assumption of normality.}

In this subsection, we assume that the population distribution is normal with unknown mean $\mu$ and unknown variance $\sigma^{2}$. Since the proposed test statistic in (6) is location free, we do not need to estimate $\mu$. But we should use an appropriate estimator for $\sigma$ in (6). We use the following estimator of $\sigma$ in (6):

$\hat{\sigma}=\sqrt{\frac{1}{n} \sum_{i=1}^{n} S_{i}^{2}}$,

where $S_{i}^{2}=\frac{1}{k} \sum_{j=1}^{k}\left(X_{[j] j i}-\bar{X}_{. i}\right)^{2}$ and $\bar{X}_{. i}=\frac{1}{k} \sum_{j=1}^{k} X_{[j] j i}$.

It is clear that $\hat{\sigma}$ is an equivariant estimator of $\sigma$ and therefore by replacing $\sigma$ in (6) by (7), the resulting test statistic (denoted by $\hat{D}_{k, n}$ ) and thus its critical values do not depend on the unknown parameters.

It is also worth mentioning that although the expected values of the order statistics have not yet been analytically obtained, these values can be readily computed by using computer programming with arbitrary precision. Thus there is no problem in computing the test statistic in practice. But unfortunately the form of the test statistic is too complicated to allow us to derive its exact distribution under the assumptions of normality and perfect ranking; therefore we derive the critical values of $\hat{D}_{k, n}$ by simulation. 
Table 1 shows the critical values of $\hat{D}_{k, n}$ under the assumption of normality for different values of $k$ and $n$ at significance level $\alpha=.05$ based on 100,000 repetitions.

Table 1. Critical values of $\hat{D}_{k, n}$ under assumption of normality at significance level $\alpha=.05$

\begin{tabular}{|r|c|r|r|r|r|r|r|r|r|}
\hline$n$ & 2 & 3 & 4 & 5 & 6 & 7 & 8 & 9 & 10 \\
\hline 1 & 14.28 & 17.40 & 34.08 & 43.78 & 58.68 & 77.70 & 94.95 & 116.32 & 134.79 \\
\hline 2 & 43.65 & 65.76 & 99.09 & 147.48 & 196.47 & 261.11 & 329.29 & 400.81 & 485.02 \\
\hline 3 & 60.62 & 115.13 & 191.55 & 286.34 & 404.89 & 535.29 & 685.08 & 839.44 & 1026.29 \\
\hline 4 & 82.36 & 176.12 & 308.29 & 468.23 & 661.51 & 892.44 & 1158.38 & 1437.86 & 1741.38 \\
\hline 5 & 107.55 & 242.96 & 440.91 & 689.04 & 979.88 & 1340.67 & 1678.79 & 2161.83 & 2609.39 \\
\hline
\end{tabular}

Remark 3: We also provide simulation-based critical values of our proposed test for the tdistribution with $\mathrm{df}=2,3,4,5,10$ (Tables 10-14 in the appendix), which can be used if the assumption of normality seems too ideal.

We used the following scenarios (the same as those in Vock and Balakrishnan, 2011):

- Concomitant. We order the values of a concomitant variable $Y$ and use that ordering for the variable of interest $X$, which has a correlation $\lambda$ with $X$.

- Fraction of random rankings: We first perfectly order each sample of $k$ simulations and then, with probability $\lambda$, we replace each order statistics with an independent new observation from the original distribution. Thus the distribution function of $X_{[i]}$ will be $F_{[i]}=(1-\lambda) F_{(i)}+\lambda F$, for $i=1, \cdots, k$, some $\lambda \in[0,1]$.

- Fraction of inverse rankings: the same as fraction of random ranking, but we use $F_{(k-i+1)}$ instead of $F$, that is the distribution function of $X_{[i]}$ will be $F_{[i]}=(1-\lambda) F_{(i)}+\lambda F_{(k-i+1)}$, for $i=1, \cdots, k$, some $\lambda \in[0,1]$.

- Fraction of neighbors: We first perfectly order each sample of $k$ simulations and then replace the $i^{\text {th }}$ ranked observation with either the $(i-1)^{\text {th }}$ or $(i+1)^{\text {th }}$ ranked observation, both with probability $\frac{\lambda}{2}$. Thus the distribution function of $X_{[i]}$ will be $F_{[i]}=\frac{\lambda}{2} F_{(i-1)}+(1-\lambda) F_{(i)}+\frac{\lambda}{2} F_{(i+1)}$, for $i=1, \cdots, k$, some $\lambda \in[0,1]$ and $F_{(0)}=F_{(1)}, F_{(k+1)}=F_{(k)}$.

The power estimates for the proposed test have been obtained based on 100,000 repetitions for different values of $\lambda,(n, k)$ and under different imperfect ranking scenarios at significance level of $\alpha=0.05$. The results (partly taken from Tables 3-6 in Vock and Balakrishnan, 2011) are reported in Tables 2-5 and visualized in figures 1-4. In these tables, bold types indicate that the test statistic achieves the maximum power among its competitors. 
Table 2. Power estimates of the tests based on $J_{k, n}, N_{k, n}, s_{k, n}, A_{k, n}, w^{*}, \hat{D}_{k, n}$ under the assumption of normality and concomitant model with correlation $\lambda$ at significance level $\alpha=0.05$

\begin{tabular}{|c|c|c|c|c|c|c|c|c|c|c|c|c|c|}
\hline$k$ & $n$ & $\begin{array}{c}\text { Test } \\
\text { Statistic }\end{array}$ & $\lambda$ & & & & & & & & & & \\
\hline & & & .5 & .55 & .60 & .65 & .70 & .75 & .80 & .85 & .90 & .95 & 1 \\
\hline 2 & 5 & $J_{k, n}$ & .3180 & .2838 & .2507 & .2215 & .1890 & .1631 & .1353 & .1092 & .0878 & .0663 & .0506 \\
\hline 2 & 5 & $N_{k, n}$ & .2468 & .2244 & .2010 & .1771 & .1530 & .1327 & .1132 & .0950 & .0791 & .0629 & .0493 \\
\hline 2 & 5 & $s_{k, n}$ & .2468 & .2244 & .2010 & .1771 & .1530 & .1327 & .1132 & .0950 & .0791 & .0629 & .0493 \\
\hline 2 & 5 & $A_{k, n}$ & .2468 & .2244 & .2010 & .1771 & .1530 & .1327 & .1132 & .0950 & .0791 & .0629 & .0493 \\
\hline 2 & 5 & $w^{*}$ & .3181 & .2839 & .2508 & .2216 & .1890 & .1632 & .1353 & .1093 & .0878 & .0663 & .0506 \\
\hline 2 & 5 & $\hat{D}_{k, n}$ & .3290 & .2938 & .2617 & .2259 & 1969 & 1685 & .1381 & .1129 & .0879 & .0677 & .0492 \\
\hline 5 & 2 & $J_{k, n}$ & .7366 & .6921 & .6367 & .5765 & .5086 & .4351 & .3529 & .2669 & .1877 & .1113 & .0503 \\
\hline 5 & 2 & $N_{k, n}$ & .6820 & .6345 & .5799 & .5202 & .4560 & .3878 & .3140 & .2386 & .1694 & .1038 & .0503 \\
\hline 5 & 2 & $s_{k, n}$ & .6982 & .6498 & .5942 & .5339 & .4672 & .3995 & .3259 & .2521 & .1742 & .1074 & .0511 \\
\hline 5 & 2 & $A_{k, n}$ & .6689 & .6213 & .5693 & .5107 & .4489 & .3826 & .3096 & .2371 & .1677 & .1037 & .0499 \\
\hline 5 & 2 & $w^{*}$ & .7480 & .6990 & .6488 & .5857 & .5188 & .4402 & .3624 & .2772 & .1924 & .1133 & .0508 \\
\hline 5 & 2 & $\hat{D}_{k, n}$ & .7603 & .7140 & .6644 & .6012 & .5328 & .4554 & .3726 & .2867 & .1974 & .1159 & .0483 \\
\hline 4 & 5 & $J_{k, n}$ & .8876 & .8454 & .7961 & .7299 & .6510 & .5577 & .4517 & .3403 & .2245 & .1252 & .0508 \\
\hline 4 & 5 & $N_{k, n}$ & .8047 & .7555 & .6930 & .6262 & .5457 & .4622 & .3715 & .2754 & .1856 & .1075 & .0506 \\
\hline 4 & 5 & $S_{k, n}$ & .8083 & .7588 & .6965 & .6307 & .5516 & .4641 & .3728 & .2756 & .1878 & .1085 & .0488 \\
\hline 4 & 5 & $A_{k, n}$ & .7827 & .7293 & .6683 & .6026 & .5244 & .4413 & .3526 & .2632 & .1791 & .1053 & .0499 \\
\hline 4 & 5 & $w^{*}$ & .8846 & .8431 & .7913 & .7281 & .6496 & .5570 & .4510 & .3367 & .2234 & .1235 & .0511 \\
\hline 4 & 5 & $\hat{D}_{k, n}$ & .8809 & .8376 & .7860 & .7193 & 6399 & .5498 & .4450 & .3303 & .2222 & .1229 & .0503 \\
\hline 5 & 4 & $J_{k, n}$ & .9439 & .9175 & .8805 & .8338 & .7639 & .6752 & .5629 & .4316 & .2856 & .1514 & .0510 \\
\hline 5 & 4 & $N_{k, n}$ & .8877 & .8504 & .7988 & .7392 & .6644 & .5770 & .4688 & .3533 & .2381 & .1282 & .0503 \\
\hline 5 & 4 & $s_{k, n}$ & .8994 & .8621 & .8158 & .7544 & .6801 & .5935 & .4822 & .3689 & .2440 & .1355 & .0507 \\
\hline 5 & 4 & $A_{k, n}$ & .8810 & .8433 & .7918 & .7316 & .6525 & .5636 & .4592 & .3471 & .2307 & .1287 & .0512 \\
\hline 5 & 4 & $W^{*}$ & .9448 & .9175 & .8792 & .8326 & .7649 & .6754 & .5646 & .4324 & .2883 & .1513 & .0494 \\
\hline 5 & 4 & $\hat{D}_{k, n}$ & .9355 & .9101 & .8711 & .8219 & .7493 & .6607 & .5495 & .4198 & .2795 & .1470 & .0490 \\
\hline
\end{tabular}


Table 3.Power estimates of the tests based on $J_{k, n}, N_{k, n}, s_{k, n}, A_{k, n}, w^{*}, \hat{D}_{k, n}$ under the assumption of normality and fraction of random rankings model with fraction $\lambda$ at significance level $\alpha=0.05$

\begin{tabular}{|c|c|c|c|c|c|c|c|c|c|c|c|c|c|}
\hline \multirow[t]{2}{*}{$k$} & $n$ & $\begin{array}{c}\text { Test } \\
\text { Statistic } \\
\end{array}$ & $\lambda$ & & & & & & & & & & \\
\hline & & & 0 & .05 & 0.1 & 0.15 & 0.2 & 0.25 & 0.3 & 0.35 & 0.4 & 0.45 & 0.5 \\
\hline 2 & 5 & $J_{k, n}$ & .0502 & .0651 & .0847 & 1077 & .1296 & .1541 & .1789 & 2102 & .2404 & 2716 & 3067 \\
\hline 2 & 5 & $N_{k, n}$ & .0501 & .0618 & .0756 & .0908 & .1092 & .1268 & .1474 & .1681 & .1925 & 2170 & 2403 \\
\hline 2 & 5 & $s_{k, n}$ & .0501. & .0618. & .0756 & .0908. & 1092 & 1268 & .1474 & .1681. & 1925 & 2170 & 2403 \\
\hline 2 & 5 & $A_{k, n}$ & .0501 & .0618 & .0756 & .0908 & .1092 & .1268 & .1474 & .1681 & .1925 & .2170 & 2403 \\
\hline 2 & 5 & $w^{*}$ & .0503 & .0652 & .0847 & .1078 & .1296 & .1541 & .1790 & .2102 & .2404 & .2717 & .3068 \\
\hline 2 & 5 & $\hat{D}_{k, n}$ & .0509 & .0692 & .0902 & .1155 & .1402 & .1675 & .1987 & .2307 & .2593 & .2942 & .3301 \\
\hline 5 & 2 & $J_{k, n}$ & .0500. & .1117 & .1758 & 2433 & .3123 & .3786 & .4481 & .5120 & .5719 & .6276 & .6775 \\
\hline 5 & 2 & $N_{k, n}$ & .0484 & .1015 & .1595 & .2199 & .2815 & .3456 & .4061 & .4653 & .5242 & .5783 & 6304 \\
\hline 5 & 2 & $s_{k, n}$ & .0500 & 1143 & .1795 & .2447 & .3120 & .3749 & .4402 & .4998 & .5545 & 6080 & .6579 \\
\hline 5 & 2 & $A_{k, n}$ & .0506 & .0987 & 1499 & .2062 & .2660 & .3245 & .3851 & .4424 & .4995 & .5530 & .6046 \\
\hline 5 & 2 & $W^{*}$ & .0518 & .1233 & 1988 & .2708 & .3443 & .4112 & .4801 & .5408 & .6017 & .6550 & .7024 \\
\hline 5 & 2 & $\hat{\mathrm{D}}_{k, n}$ & .0482 & 1349. & 2201 & .3024 & 3762 & .4472 & .5146 & .5764 & .6321 & 68824. & .7308 \\
\hline & & & & & & & & & & & & & \\
\hline 4 & 5 & $J_{k, n}$ & .0504 & 1204 & 2047 & .2959 & .3934 & .4912 & .5767 & .6572 & .7291 & $\begin{array}{l}.7873 \\
7\end{array}$ & .8376 \\
\hline 4 & 5 & $N_{k, n}$ & .0500 & 1043 & .1701 & .2469 & .3260 & .4071 & .4854 & .5629 & .6332 & .7001 & 7588 \\
\hline 4 & 5 & $s_{k, n}$ & .0502 & 1137 & 1891 & .2712 & .3529 & .4364 & .5179 & .5923 & .6594 & .7231 & 7768 \\
\hline 4 & 5 & $A_{k, n}$ & .0504 & .0985 & 1584 & .2276 & .2960 & .3764 & .4536 & .5264 & .5976 & 6637 & .7200 \\
\hline 4 & 5 & $w^{*}$ & .0488 & .1312 & .2260 & .3257 & .4238 & .5173 & .6041 & .6817 & .7468 & .8042 & .8505 \\
\hline 4 & 5 & $\hat{D}_{k, n}$ & .0514 & .1639 & .2743 & .38156 & .4808 & .5649 & .6470 & .7161 & .7754 & .8236 & .8661 \\
\hline 5 & 4 & $J_{k, n}$ & .0483 & .1412 & .2480 & .3596 & .4700 & .5716 & .6635 & .7425 & .8061 & .8567 & .8966 \\
\hline 5 & 4 & $N_{k, n}$ & .0492 & 1196 & .2057 & .3007 & .3969 & .4900 & .5775 & .6535 & .7248 & .7845 & .8362 \\
\hline 5 & 4 & $s_{k, n}$ & .0488 & .1418 & .2462 & .3485 & .4492 & .5416 & .6294 & .7022 & .7610 & 8202 & .8618 \\
\hline 5 & 4 & $A_{k, n}$ & .0488 & 1141 & 1908 & 2814 & .3732 & .4650 & .5513 & .6300 & .6998 & .7650 & 8157 \\
\hline 5 & 4 & $w^{*}$ & .0488 & 1644 & .2814 & .3987 & .5144 & .6116 & .6962 & .7706 & .8260 & .8725 & 9090 \\
\hline 5 & 4 & $\hat{D}_{k, n}$ & .0510 & .1972 & .3338 & .4598 & .5601 & .6530 & .7295 & .7936 & .8469 & .8880 & .9179 \\
\hline
\end{tabular}


Table 4. Power estimates of the tests based on $J_{k, n}, N_{k, n}, s_{k, n}, A_{k, n}, W^{*}, \hat{D}_{k, n}$ under the assumption of normality and fraction of inverse rankings model with fraction $\lambda$ at significance level $\alpha=0.05$

\begin{tabular}{|c|c|c|c|c|c|c|c|c|c|c|c|c|c|}
\hline \multirow[t]{2}{*}{$k$} & \multirow[t]{2}{*}{$n$} & \multirow[t]{2}{*}{$\begin{array}{c}\text { Test } \\
\text { Statistic }\end{array}$} & \multicolumn{2}{|l|}{$\lambda$} & \multirow[b]{2}{*}{0.1} & \multirow{2}{*}{0.15} & \multirow{2}{*}{0.2} & \multirow{2}{*}{0.25} & \multirow{2}{*}{0.3} & & \multirow{2}{*}{0.4} & \multirow{2}{*}{0.45} & \multirow{2}{*}{0.5} \\
\hline & & & 0 & .05 & & & & & & & & & \\
\hline 2 & 5 & $J_{k, n}$ & .0502 & .0859 & .1237 & .1819 & .2421 & .3061 & .3776 & .4535 & .5191 & .5905 & 6590 \\
\hline 2 & \multirow{2}{*}{$\frac{5}{5}$} & $N_{k, n}$ & .0501 & .0760 & .1082 & .1437 & .1892 & .2405 & .2930 & .3475 & .4051 & 4650 & .5301 \\
\hline 2 & & $s_{k, n}$ & .0501. & .0760 & 1082 & 1437 & 1892 & 2405 & 2930 & .3475 & .4051 & 4650 & .5301 \\
\hline 2 & \multirow{2}{*}{$\frac{5}{5}$} & $A_{k, n}$ & .0501 & .0760 & .1082 & .1437 & .1892 & .2405 & .2930 & .3475 & .4051 & 4650 & .5301 \\
\hline 2 & & $w^{*}$ & .0503 & .0859 & .1237 & .1820 & .2422 & .3061 & .3777 & .4535 & .5192 & .5905 & .6590 \\
\hline 2 & 5 & $\hat{D}_{k, n}$ & .0499. & .0901 & .1421 & .1960 & .2630 & .3294 & 3998 & .4702 & .5386 & .6080 & .6690 \\
\hline 5 & 2 & $J_{k, n}$ & .0500 & .1757 & .3014 & .4224 & .5307 & .6275 & .7114 & .7842 & .8419 & 8878 & .9246 \\
\hline 5 & 2 & $N_{k, n}$ & .0484 & .1589 & .2745 & .3873 & .4888 & .5856 & .6740 & .7482 & .8114 & 8620 & .9029 \\
\hline 5 & 2 & $s_{k, n}$ & .0500 & .1890 & .3183 & .4402 & .5452 & .6378 & .7191 & .7876 & .8439 & .8850 & .9205 \\
\hline 5 & 2 & $A_{k, n}$ & .0506 & 1499 & .2524 & .3544 & .4520 & .5477 & .6317 & .7052 & .7711 & 8264 & .8729 \\
\hline 5 & 2 & $W^{*}$ & .0518 & 2045 & .3452 & .4722 & .5799 & .6770 & .7518 & .8172 & .8678 & 9075 & .9380 \\
\hline 5 & 2 & $\hat{D}_{k, n}$ & .0496 & .2218 & .3766 & .5027 & .6122 & .7019 & .7766 & 8372 & .8827 & .9182 & .9448 \\
\hline 4 & 5 & $J_{k, n}$ & .0504 & 2020 & .3754 & .5374 & .6761 & .7866 & .8675 & .9226 & .9572 & .9768 & .9893 \\
\hline 4 & 5 & $N_{k, n}$ & .0500 & .1685 & .3128 & .4615 & .5961 & .7150 & .8078 & .8761 & .9241 & .9556 & .9776 \\
\hline 4 & 5 & $s_{k, n}$ & .0502 & .2007 & .3610 & .5178 & .6499 & .7566 & .8403 & .8994 & .9397 & .9659 & .9816 \\
\hline 4 & 5 & $A_{k, n}$ & .0504 & .1516 & .2786 & .4129 & .5423 & .6616 & .7573 & .8348 & .8921 & .9358 & .9629 \\
\hline 4 & 5 & $w^{*}$ & .0488 & .2377 & .4283 & .5933 & .7246 & .8221 & .8913 & .9380 & .9666 & .9828 & .9914 \\
\hline 4 & 5 & $\hat{D}_{k, n}$ & .0505 & .3108 & .5166 & .6712 & .7855 & .8637 & .9192 & .9543 & .9747 & .9869 & .9939 \\
\hline 5 & 4 & $J_{k n}$ & 0483 & .2398 & 4356 & 6079 & 7410 & 8387 & 9052 & 9481 & 9731 & 9880 & 9944 \\
\hline 5 & 4 & $N_{k, n}$ & .0492 & .2006 & .3698 & .5329 & .6747 & .7828 & .8600 & .9180 & .9547 & .9750 & .9878 \\
\hline 5 & 4 & $s_{k, n}$ & .0488 & 2565 & .4526 & .6173 & .7398 & .8356 & .8994 & .9428 & .9686 & .9848 & .9926 \\
\hline 5 & 4 & $A_{k, n}$ & .0488 & .1834 & .3381 & .4905 & .6281 & .7426 & .8280 & .8924 & .9361 & .9640 & .9813 \\
\hline 5 & 4 & $w^{*}$ & .0495 & .2941 & .5075 & .6740 & .7920 & .8761 & .9295 & .9625 & .9811 & .9910 & .9958 \\
\hline 5 & 4 & $\hat{D}_{k, n}$ & .0494 & .3453 & .5674 & .7231 & .8288 & .8982 & .9414 & .9696 & .9841 & .9923 & .9967 \\
\hline
\end{tabular}


Table 5. Power estimates of the tests based on $J_{k, n}, N_{k, n}, s_{k, n}, A_{k, n}, w^{*}, \hat{D}_{k, n}$ under the assumption of normality and fraction of neighbors model with fraction $\lambda$ at significance level $\alpha=0.05$

\begin{tabular}{|c|c|c|c|c|c|c|c|c|c|c|c|c|c|}
\hline \multirow[t]{2}{*}{$k$} & & \multirow[t]{2}{*}{$\begin{array}{c}\text { Test } \\
\text { Statistic }\end{array}$} & \multicolumn{2}{|l|}{$\lambda$} & \multirow[b]{2}{*}{0.2} & \multirow{2}{*}{0.3} & \multirow{2}{*}{0.4} & \multirow{2}{*}{0.5} & \multirow{2}{*}{0.6} & \multirow{2}{*}{0.7} & \multirow{2}{*}{0.8} & \multirow{2}{*}{0.9} & \multirow{2}{*}{1} \\
\hline & & & 0 & 0.1 & & & & & & & & & \\
\hline 2 & 5 & $J_{k, n}$ & .0502 & .0851 & .1285 & .1821 & .2413 & .3054 & .3754 & .4466 & .5179 & .5894 & .6562 \\
\hline$\frac{2}{2}$ & 5 & $N_{k, n}$ & .0501 & .0758 & .1079 & .1463 & .1917 & .2387 & .2910 & .3490 & .4064 & 4698 & .5269 \\
\hline 2 & 5 & $s_{k, n}$ & .0501. & .0758 & 1079 & 1463 & 1917. & 2387 & 2910. & .3490 & .4064 & 4698 & .5269 \\
\hline 2 & \multirow{2}{*}{$\frac{5}{5}$} & $A_{k, n}$ & .0501 & .0758 & .1079 & .1463 & .1917 & .2387 & .2910 & .3490 & .4064 & .4698 & .5269 \\
\hline 2 & & $w^{*}$ & .0503 & .0852 & .1285 & .1822 & .2413 & .3055 & .3755 & .4467 & .5179 & .5895 & .6562 \\
\hline 2 & 5 & $\hat{D}_{k, n}$ & .0512. & .0897 & .1425. & .1958 & 2596 & .3274 & .4006 & .4701 & .5395 & .6072 & 6734 \\
\hline 5 & 2 & $J_{k, n}$ & .0500 & .0694 & .0930 & .1189 & 1437 & 1750 & 2046 & 2418 & .2777 & 3148 & 3505 \\
\hline 5 & 2 & $N_{k, n}$ & .0484 & .0673 & .0861 & .1081 & .1346 & .1562 & .1873 & .2140 & .2464 & .2770 & .3086 \\
\hline 5 & 2 & $s_{k, n}$ & .0500 & .0660 & .0868 & .1080 & .1309 & .1551 & .1792 & .2098 & .2334 & .2666 & .2930 \\
\hline 5 & 2 & $A_{k, n}$ & .0506 & .0681 & .0881 & .1117 & .1377 & .1647 & .1940 & .2248 & .2543 & 2919 & .3299 \\
\hline 5 & 2 & $W^{*}$ & .0518 & .0703 & .0916 & .1168 & .1420 & .1698 & 2001 & .2317 & .2616 & .2973 & .3291 \\
\hline 5 & 2 & $\hat{D}_{k, n}$ & .0497 & .0708 & .0921 & .1168 & .1436 & .1701 & .1961 & .2282 & .2604 & .2892 & .3232 \\
\hline 4 & 5 & $J_{k, n}$ & .0504 & .0866 & .1341 & .1892 & .2538 & 3229 & .3980 & .4762 & .5509 & .6254 & 6939 \\
\hline 4 & 5 & $N_{k, n}$ & .0500 & .0798 & .1178 & .1592 & .2079 & .2634 & .3205 & .3818 & .4444 & .5066 & .5674 \\
\hline 4 & 5 & $s_{k, n}$ & .0502 & .0765 & .1133 & .1535 & .1998 & .2477 & .3010 & .3592 & .4154 & .4728 & .5274 \\
\hline 4 & 5 & $A_{k, n}$ & .0504 & .0771 & .1136 & .1580 & .2064 & .2615 & .3224 & .3836 & .4482 & .5120 & .5748 \\
\hline 4 & 5 & $w^{*}$ & .0488 & .0842 & .1292 & .1816 & .2423 & .3062 & .3730 & .4428 & .5106 & .5796 & .6430 \\
\hline 4 & 5 & $\hat{D}_{k, n}$ & .0499 & .0863 & .1275 & .1779 & .2289 & .2848 & .3440 & .4088 & .4669 & .5315 & .5900 \\
\hline 5 & 4 & $J_{k n}$ & 0483 & 0784 & 1190 & 1635 & 2159 & 2750 & 3349 & 4004 & 4659 & 5399 & 5949 \\
\hline 5 & 4 & $N_{k, n}$ & .0492 & .0742 & .1045 & .1407 & .1805 & .2258 & .2718 & .3214 & .3775 & .4298 & .4812 \\
\hline 5 & 4 & $s_{k, n}$ & .0488 & .0744 & .1029 & .1364 & .1750 & .2172 & .2608 & .3058 & .3555 & .4065 & .4530 \\
\hline 5 & 4 & $A_{k, n}$ & .0488 & .0750 & .1060 & .1434 & .1867 & .2333 & .2829 & .3386 & .3955 & 4510 & .5105 \\
\hline 5 & 4 & $w^{*}$ & .0495 & .0804 & .1136 & .1569 & .2048 & .2557 & .3103 & .3695 & .4285 & .4858 & .5485 \\
\hline 5 & 4 & $\hat{D}_{k, n}$ & .0494 & .0796 & .1161 & .1525 & 1965 & .2362 & .2899 & .3321 & .3841 & .4353 & .4857 \\
\hline
\end{tabular}

The results based on the concomitant model are represented in Table 2. It is clear from this table that none of tests dominates the others in terms of their powers. The tests based on $J_{k, n}, W^{*}$ and $\hat{D}_{k, n}$ are quite competitive. For $(k, n)=(2,5),(5,2), \hat{D}_{k, n}$ (the proposed test) is the best, $J_{k, n}$ is the best when $(k, n)=(4,5)$. For $(k, n)=(5,4), J_{k, n}$ and $W^{*}$ are quite competitive and the differences between their powers are not considerable. 

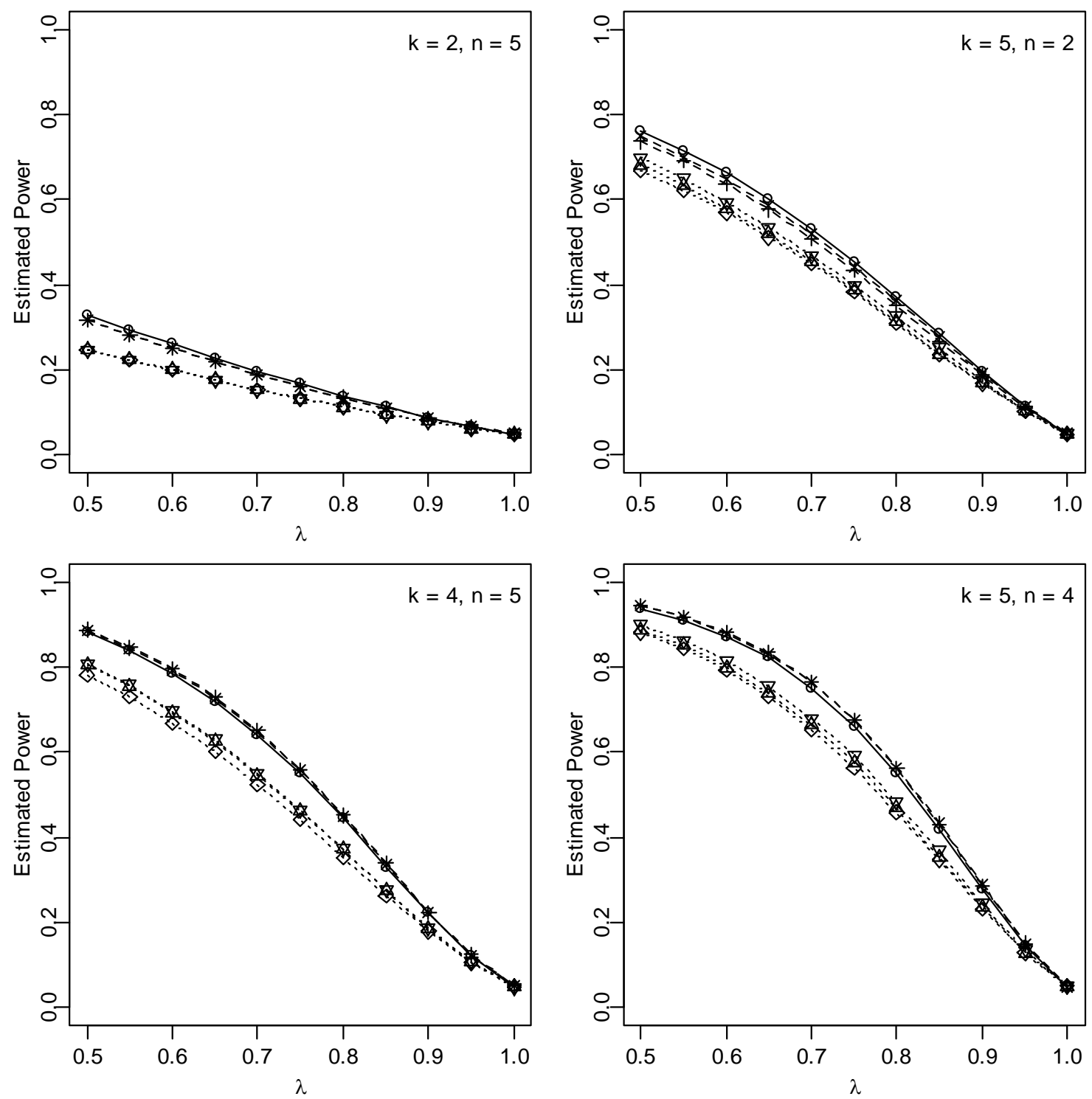

Figure 1. Visualized estimated powers of tests based on $J_{k, n}($ represented by +$), N_{k, n}$ (represented by $\Delta), S_{k, n}$ (represented by $\nabla$ ), $A_{k, n}$ (represented by $\left.\diamond\right), W^{*}$ (represented by $\times), D_{k, n}$ (represented by $\bigcirc$ ) in Table 2 under the assumption of normality and concomitant model with correlation $\lambda$ at significance level $\alpha=0.05$ 

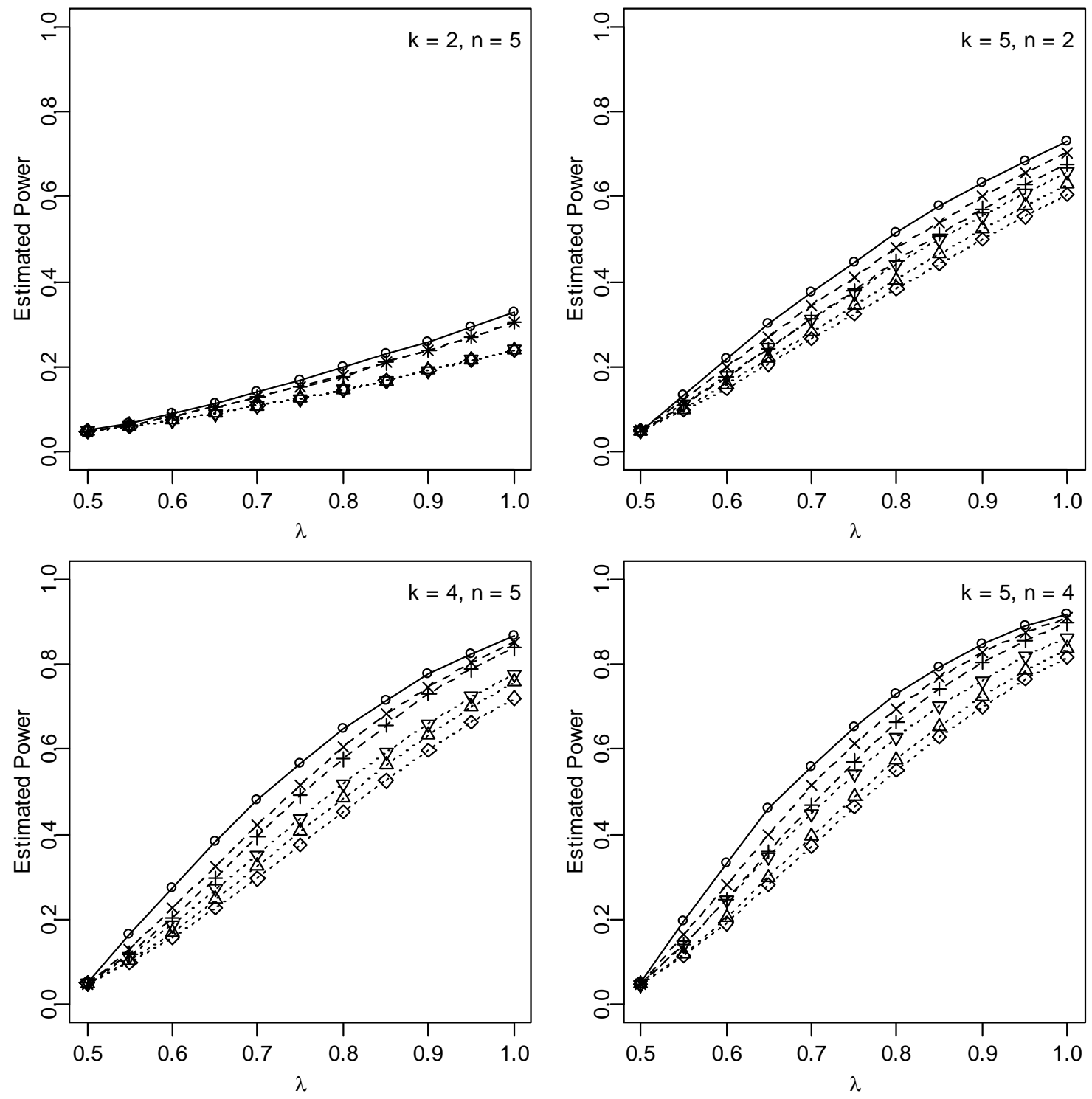

Figure 2. Visualized estimated powers of tests based on $J_{k, n}($ represented by +$), N_{k, n}$ (represented by $\Delta$ ), $S_{k, n}$ (represented by $\left.\nabla\right), A_{k, n}$ (represented by $\left.\diamond\right), W^{*}$ (represented by $\times), D_{k, n}$ (represented by $\bigcirc$ ) in Table 3 under the assumption of normality and fraction of random rankings model with fraction $\lambda$ at significance level $\alpha=0.05$. 

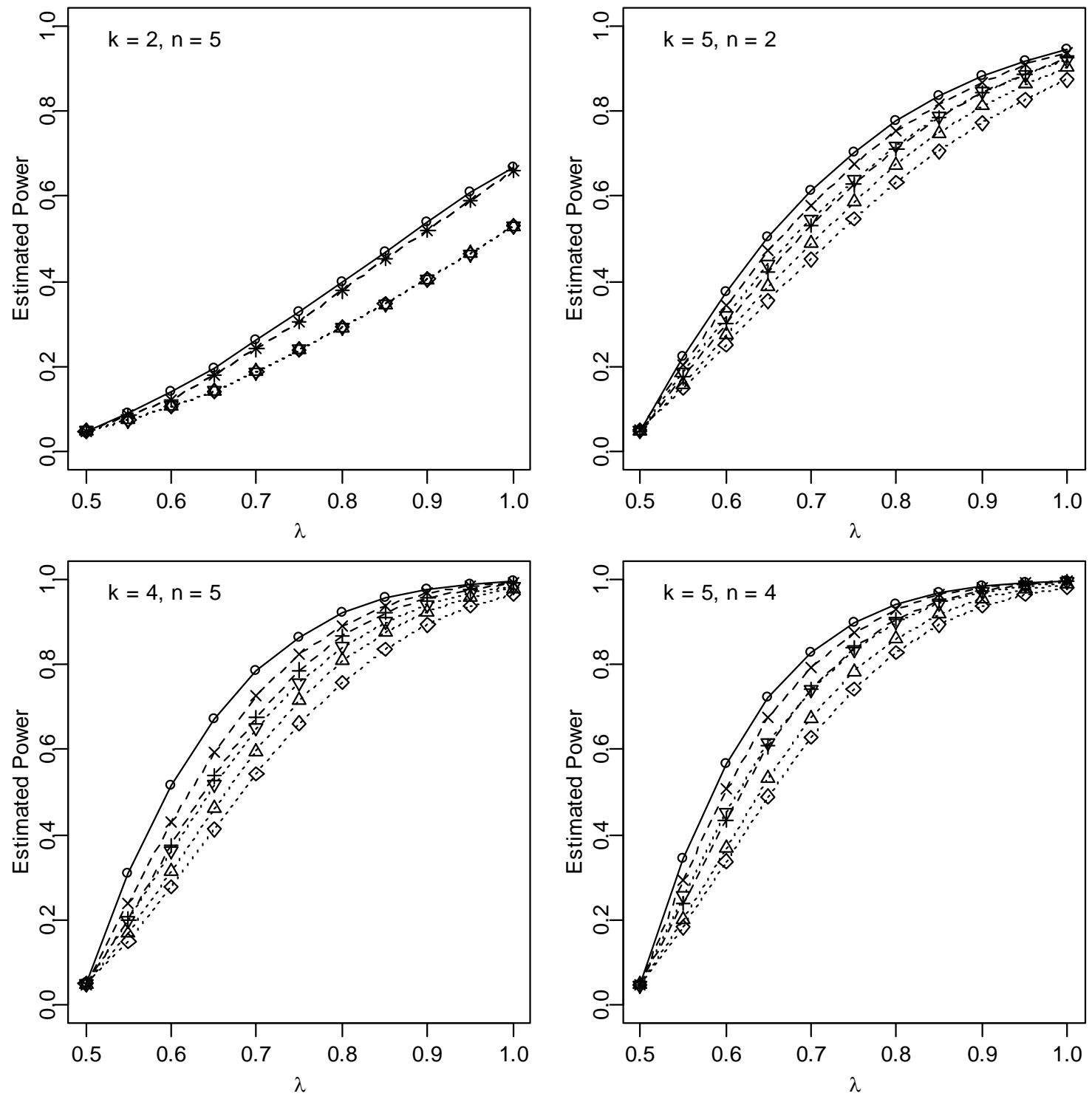

Figure 3. Visualized estimated powers of tests based on $J_{k, n}\left(\right.$ represented by + ), $N_{k, n}$ (represented by $\Delta), S_{k, n}$ (represented by $\left.\nabla\right), A_{k, n}($ represented by $\diamond), W^{*}$ (represented by $\times), D_{k, n}$ (represented by $\bigcirc$ ) in Table 4 under the assumption of normality and fraction of inverse rankings model with fraction $\lambda$ at significance level $\alpha=0.05$. 

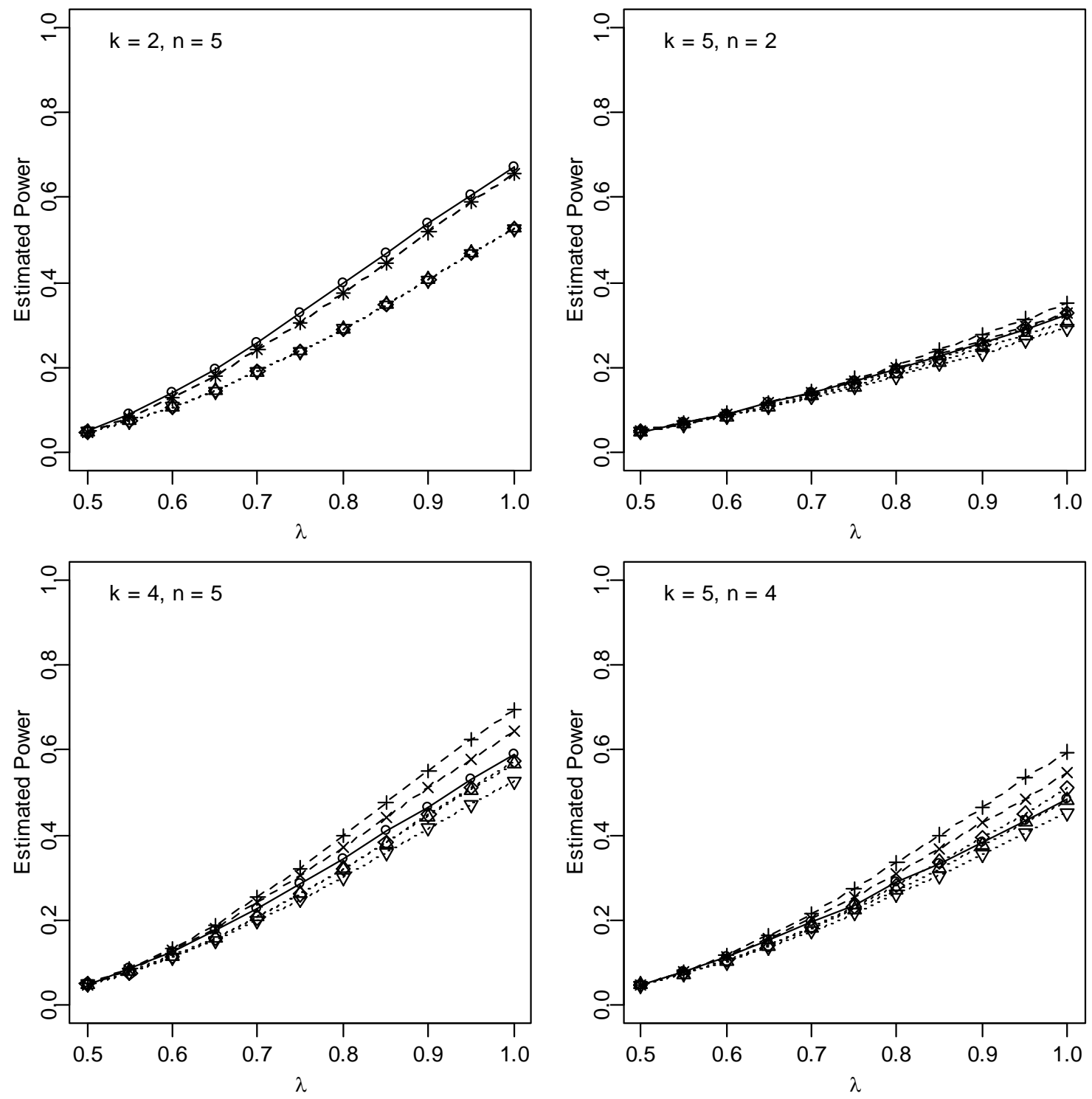

Figure 4. Visualized estimated powers of tests based on $J_{k, n}\left(\right.$ represented by + ), $N_{k, n}$ (represented by $\Delta), S_{k, n}$ (represented by $\left.\nabla\right), A_{k, n}($ represented by $\diamond), W^{*}$ (represented by $\times), D_{k, n}$ (represented by $\bigcirc$ ) in Table 4 under the assumption of normality and fraction of neighbors model with fraction $\lambda$ at significance level $\alpha=0.05$ 
Tables 3 and 4 contain empirical powers of the tests under fraction random rankings and fraction inverse ranking models, respectively. Obviously, under these imperfect ranking models, the power of the proposed test is consistently better than all of its competitors in all values of $\lambda$, sample sizes and numbers of cycles considered.

The results are more in favor of $J_{k, n}$ than other tests when the imperfect ranking model is fraction of neighbors (Table 5). For $(k, n)=(2,5)$, the proposed test is best, while in other cases, the test based on $J_{k, n}$ has the greatest power (except for $\lambda=.1$ ). It is also worth mentioning that the power of tests based on $\hat{D}_{k, n}$ and $J_{k, n}$ are quite competitive when $(k, n)=(5,2)$ for $\lambda \leq .6$ and when $\lambda$ tends to 1 , the difference between powers of tests based on $\hat{D}_{k, n}$ and $J_{k, n}$ increases.

\subsection{Empirical power comparison under assumption of exponentiality}

In this subsection, we assume that the population distribution is exponential with an unknown mean $\sigma$. The following theorem will help us in the construction of the test statistic.

Theorem 2. Let $X_{(1)}, X_{(2)}, \cdots, X_{(k)}$ be order statistics of a sample of size $k$ from $a$ standard exponential distribution, then

$$
\begin{aligned}
& \text { I) } E\left(X_{(i)}\right)=c_{i, k}=\sum_{j=1}^{i} \frac{1}{k-j+1} ; \\
& I I) \operatorname{Var}\left(X_{(i)}\right)=d_{i, k}=\sum_{j=1}^{i} \frac{1}{(k-j+1)^{2}} .
\end{aligned}
$$

The proof follows from equation (2.1) in Sengupta and Mukhuti (2006).

Now, by the above theorem and Lagrangian method, it is not hard to obtain the BLUE of $\sigma$ based on BRSS. The estimate has the following form:

$$
\hat{\sigma}=\sum_{i=1}^{n} \sum_{j=1}^{k} \frac{c_{j, k}}{n d_{j, k}\left(\sum_{j=1}^{k} \frac{c_{j, k}^{2}}{d_{j, k}}\right)} X_{[j] j i}
$$

It is clear that $\hat{\sigma}$ is an equivariant estimator of $\sigma$ and therefore by replacing $\sigma$ in (6) by (8), the resulting test statistic $\left(\hat{D}_{k, n}\right)$ and thus its critical values do not depend on the unknown parameter.

Although the test statistic can be readily computed via theorem 2, the form of it is too complicated to allow us to derive its exact distribution under the assumptions of exponentiality and perfect ranking. Therefore we derive critical values of $\hat{D}_{k, n}$ by simulation. 
Table 6 shows the critical values of $\hat{D}_{k, n}$ under the assumption of exponentiality for different values of $k$ and $n$ at significance level $\alpha=.05$ based on 100,000 repetitions.

Table 6. Critical values of $\hat{D}_{k, n}$ under the assumption of exponentiality at significance level $\alpha=.05$

\begin{tabular}{|c|c|c|c|c|c|c|c|c|c|}
\hline$k$ & 2 & 3 & 4 & 5 & 6 & 7 & 8 & 9 & 10 \\
\hline 1 & 7.84 & 12.56 & 25.49 & 36.12 & 51.53 & 67.80 & 86.71 & 106.36 & 128.53 \\
\hline 2 & 20.21 & 49.13 & 84.28 & 132.84 & 188.91 & 248.24 & 319.28 & 398.30 & 477.91 \\
\hline 3 & 37.90 & 97.53 & 178.30 & 273.46 & 395.01 & 525.55 & 673.86 & 846.18 & 1044.74 \\
\hline 4 & 59.96 & 154.71 & 290.60 & 451.87 & 651.97 & 893.15 & 1153.49 & 1444.80 & 1790.55 \\
\hline 5 & 85.79 & 223.59 & 425.01 & 679.49 & 982.23 & 1338.39 & 1731.28 & 2220.81 & 2676.26 \\
\hline
\end{tabular}

We used fraction of random rankings, fraction of inverse rankings and fraction of neighbors scenarios (as defined in Subsection 3.1) for imperfect ranking.

The power estimates for the proposed test have been obtained by 100,000 repetitions for different values of $\lambda,(n, k)$ and under different imperfect ranking scenarios at significance level of $\alpha=0.05$. The results (again partly taken from Tables 3-6 in Vock and Balakrishnan, 2011) are tabulated in Tables 7-9 and visualized in Figures 5-7. In these tables, bold types indicate that the test statistic achieves the maximum power among its competitors. 
Table 7. Power estimates of the tests based on $J_{k, n}, N_{k, n}, s_{k, n}, A_{k, n}, w^{*}, \hat{D}_{k, n}$ under the assumption of exponentiality and fraction of random rankings model with fraction $\lambda$ at significance level $\alpha=0.05$

\begin{tabular}{|c|c|c|c|c|c|c|c|c|c|c|c|c|c|}
\hline \multirow[t]{2}{*}{$k$} & $n$ & $\begin{array}{l}\text { Test } \\
\text { Statistic }\end{array}$ & $\lambda$ & & & & & & & & & & \\
\hline & & & 0 & .05 & 0.1 & 0.15 & 0.2 & 0.25 & 0.3 & 0.35 & 0.4 & 0.45 & 0.5 \\
\hline 2 & 5 & $J_{k, n}$ & .0502 & .0651 & .0847 & .1077 & .1296 & 1541 & .1789 & .2102 & .2404 & 2716 & 3067 \\
\hline 2 & 5 & $N_{k, n}$ & .0501 & .0618 & .0756 & .0908 & .1092 & .1268 & .1474 & .1681 & .1925 & 2170 & 2403 \\
\hline 2 & 5 & $s_{k, n}$ & .0501. & .0618. & .0756 & .0908. & 1092 & . 1268 & .1474 & . 1681 & 1925 & 2170 & 2403 \\
\hline 2 & 5 & $A_{k, n}$ & .0501 & .0618 & .0756 & .0908 & .1092 & .1268 & .1474 & .1681 & .1925 & .2170 & 2403 \\
\hline 2 & 5 & $w^{*}$ & .0503 & .0652 & .0847 & .1078 & .1296 & .1541 & .1790 & .2102 & .2404 & .2717 & 3068 \\
\hline 2 & 5 & $\hat{D}_{k, n}$ & .0492 & .0710 & .0967 & .1227 & 1511 & .1788 & 2085. & .2387 & 2713 & .3036 & .3382 \\
\hline 5 & 2 & $J_{k, n}$ & .0500 & .1117 & .1758 & .2433 & .3123 & .3786 & .4481 & .5120 & .5719 & .6276 & .6775 \\
\hline 5 & 2 & $N_{k, n}$ & .0484 & .1015 & .1595 & .2199 & .2815 & .3456 & .4061 & .4653 & .5242 & .5783 & .6304 \\
\hline 5 & 2 & $s_{k, n}$ & .0500 & .1143 & .1795 & .2447 & .3120 & .3749 & .4402 & .4998 & .5545 & .6080 & 6579 \\
\hline 5 & 2 & $A_{k, n}$ & .0506 & .0987 & .1499 & .2062 & .2660 & .3245 & .3851 & .4424 & .4995 & 5530 & .6046 \\
\hline 5 & 2 & $W^{*}$ & .0518 & .1233 & .1988 & .2708 & .3443 & .4112 & .4801 & .5408 & .6017 & .6550 & .7024 \\
\hline 5 & 2 & $\hat{\mathrm{D}}_{k, n}$ & .0503 & .1358 & 2182 & 2979 & .3793 & .4458 & .5083 & .5703 & .6293 & .6798 & .7276 \\
\hline 4 & 5 & $J_{k, n}$ & .0504 & 1204 & .2047 & .2959 & .3934 & 4912 & .5767 & .6572 & .7291 & .7873 & .8376 \\
\hline 4 & 5 & $N_{k, n}$ & .0500 & 1043 & .1701 & .2469 & .3260 & .4071 & .4854 & .5629 & .6332 & .7001 & .7588 \\
\hline 4 & 5 & $s_{k, n}$ & .0502 & .1137 & .1891 & .2712 & .3529 & .4364 & .5179 & .5923 & .6594 & .7231 & .7768 \\
\hline 4 & 5 & $A_{k, n}$ & .0504 & .0985 & .1584 & .2276 & .2960 & .3764 & .4536 & .5264 & .5976 & .6637 & .7200 \\
\hline 4 & 5 & $w^{*}$ & .0488 & .1312 & .2260 & .3257 & .4238 & .5173 & .6041 & .6817 & .7468 & .8042 & .8505 \\
\hline 4 & 5 & $\hat{D}_{k, n}$ & .0510 & .1599 & 2697 & .3788 & .4732 & .5594 & .6380 & .7086 & .7680 & .8179 & .8568 \\
\hline 5 & 4 & $J_{k n}$ & 0483 & 1412 & 2480 & 3596 & 4700 & 5716 & 6635 & .7425 & 8061 & 8567 & 8966 \\
\hline 5 & 4 & $N_{k, n}$ & .0492 & .1196 & .2057 & .3007 & .3969 & .4900 & .5775 & .6535 & .7248 & .7845 & .8362 \\
\hline 5 & 4 & $s_{k, n}$ & .0488 & .1418 & .2462 & .3485 & .4492 & .5416 & .6294 & .7022 & .7610 & .8202 & .8618 \\
\hline 5 & 4 & $A_{k, n}$ & .0488 & .1141 & .1908 & .2814 & .3732 & .4650 & .5513 & .6300 & .6998 & .7650 & .8157 \\
\hline 5 & 4 & $w^{*}$ & .0488 & 1644 & .2814 & .3987 & .5144 & .6116 & .6962 & .7706 & .8260 & .8725 & .9090 \\
\hline 5 & 4 & $\hat{\mathrm{D}}_{k, n}$ & .0512 & .1968 & .3294 & .4489 & .5585 & .6461 & .7251 & .7886 & .8401 & .8813 & .9131 \\
\hline
\end{tabular}


Table 8. Power estimates of the tests based on $J_{k, n}, N_{k, n}, s_{k, n}, A_{k, n}, w^{*}, \hat{D}_{k, n}$ under the assumption of exponentiality and fraction of inverse rankings model with fraction $\lambda$ at significance level $\alpha=0.05$

\begin{tabular}{|c|c|c|c|c|c|c|c|c|c|c|c|c|c|}
\hline \multirow[t]{2}{*}{ k } & \multirow[t]{2}{*}{1} & \multirow[t]{2}{*}{$\begin{array}{l}\text { Test } \\
\text { Statistic }\end{array}$} & \multicolumn{2}{|l|}{$\lambda$} & 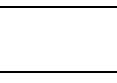 & & & & 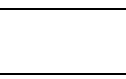 & & & & \multirow{2}{*}{0.5} \\
\hline & & & 0 & .05 & 0.1 & 0.15 & 0.2 & 0.25 & 0.3 & 0.35 & 0.4 & 0.45 & \\
\hline 2 & 5 & $J_{k, n}$ & .0502 & .0859 & .1237 & .1819 & .2421 & .3061 & .3776 & .4535 & .5191 & 5905 & .6590 \\
\hline 2 & 5 & $N_{k, n}$ & .0501 & .0760 & .1082 & .1437 & .1892 & .2405 & .2930 & .3475 & .4051 & 4650 & 5301 \\
\hline 2 & 5 & $s_{k, n}$ & .0501 & .0760 & .1082 & .1437 & .1892 & .2405 & .2930 & .3475 & .4051 & .4650 & .5301 \\
\hline 2 & 5 & $A_{k, n}$ & .0501 & .0760 & .1082 & .1437 & .1892 & .2405 & .2930 & .3475 & .4051 & .4650 & .5301 \\
\hline 2 & 5 & $w^{*}$ & .0503 & .0859 & .1237 & .1820 & .2422 & .3061 & .3777 & .4535 & .5192 & .5905 & .6590 \\
\hline 2 & 5 & $\hat{D}_{k, n}$ & .0494 & .0945 & 1491 & 2086 & 2709 & .3387 & .4039 & .4747 & .5400 & .6043 & .6657 \\
\hline 5 & 2 & $J_{k, n}$ & .0500 & 1757 & .3014 & .4224 & .5307 & .6275 & .7114 & .7842 & .8419 & 8878 & 9246 \\
\hline 5 & 2 & $N_{k, n}$ & .0484 & 1589 & .2745 & .3873 & .4888 & .5856 & .6740 & .7482 & .8114 & 8620 & .9029 \\
\hline 5 & 2 & $s_{k, n}$ & .0500 & .1890 & .3183 & .4402 & .5452 & .6378 & .7191 & .7876 & .8439 & .8850 & .9205 \\
\hline 5 & 2 & $A_{k, n}$ & .0506 & 1499 & .2524 & .3544 & .4520 & .5477 & .6317 & .7052 & .7711 & 8264 & .8729 \\
\hline 5 & 2 & $W^{*}$ & .0518 & .2045 & .3452 & .4722 & .5799 & .6770 & .7518 & .8172 & .8678 & .9075 & .9380 \\
\hline 5 & 2 & $\hat{D}_{k, n}$ & .0500 & .2226 & .3757 & .5019 & .6123 & .7024 & .7764 & .8377 & .8816 & .9187 & .9458 \\
\hline 4 & 5 & $J_{k, n}$ & .0504 & 2020 & .3754 & .5374 & .6761 & .7866 & .8675 & .9226 & .9572 & .9768 & .9893 \\
\hline 4 & 5 & $N_{k, n}$ & .0500 & .1685 & .3128 & .4615 & .5961 & .7150 & .8078 & .8761 & .9241 & .9556 & .9776 \\
\hline 4 & 5 & $s_{k, n}$ & .0502 & .2007 & .3610 & .5178 & .6499 & .7566 & .8403 & .8994 & .9397 & .9659 & .9816 \\
\hline 4 & 5 & $A_{k, n}$ & .0504 & .1516 & .2786 & .4129 & .5423 & .6616 & .7573 & .8348 & .8921 & .9358 & .9629 \\
\hline 4 & 5 & $w^{*}$ & .0488 & .2377 & .4283 & .5933 & .7246 & .8221 & .8913 & .9380 & .9666 & .9828 & .9914 \\
\hline 4 & 5 & $\hat{D}_{k, n}$ & .0512 & .2964 & .4991 & .6566 & .7736 & .8561 & .9124 & .9498 & .9716 & .9855 & .9931 \\
\hline 5 & 4 & $J_{k n}$ & 0483 & .2398 & 4356 & 6079 & 7410 & 8387 & 9052 & 9481 & 9731 & 9880 & 9944 \\
\hline 5 & 4 & $N_{k, n}$ & .0492 & .2006 & .3698 & .5329 & .6747 & .7828 & .8600 & .9180 & .9547 & .9750 & .9878 \\
\hline 5 & 4 & $s_{k, n}$ & .0488 & 2565 & .4526 & .6173 & .7398 & .8356 & .8994 & .9428 & .9686 & .9848 & .9926 \\
\hline 5 & 4 & $A_{k, n}$ & .0488 & .1834 & .3381 & .4905 & .6281 & .7426 & .8280 & .8924 & .9361 & .9640 & .9813 \\
\hline 5 & 4 & $w^{*}$ & .0495 & .2941 & .5075 & .6740 & .7920 & .8761 & .9295 & .9625 & .9811 & 9910 & .9958 \\
\hline 5 & 4 & $\hat{\mathrm{D}}_{k, n}$ & .0503 & .3425 & .5623 & .7178 & .8264 & .8976 & .9410 & .9678 & .9843 & .9919 & .9966 \\
\hline
\end{tabular}


Table 9. Power estimates of the tests based on $J_{k, n}, N_{k, n}, s_{k, n}, A_{k, n}, w^{*}, \hat{D}_{k, n}$ under the assumption of exponentiality and fraction of neighbors model with fraction $\lambda$ at significance level $\alpha=0.05$

\begin{tabular}{|c|c|c|c|c|c|c|c|c|c|c|c|c|c|}
\hline \multirow[t]{2}{*}{ k } & $n$ & $\begin{array}{l}\text { Test } \\
\text { Statistic }\end{array}$ & $\lambda$ & & & & & & & & & & \\
\hline & & & 0 & 0.1 & 0.2 & 0.3 & 0.4 & 0.5 & 0.6 & 0.7 & 0.8 & 0.9 & 1 \\
\hline 2 & 5 & $J_{k, n}$ & .0502 & .0851 & .1285 & .1821 & .2413 & .3054 & .3754 & .4466 & .5179 & .5894 & .6562 \\
\hline 2 & 5 & $N_{k, n}$ & .0501 & .0758 & .1079 & .1463 & 1917 & .2387 & .2910 & .3490 & .4064 & .4698 & .5269 \\
\hline 2 & 5 & $s_{k, n}$ & .0501 & .0758 & .1079 & .1463 & .1917 & .2387 & .2910 & .3490 & .4064 & 4698 & .5269 \\
\hline 2 & 5 & $A_{k, n}$ & .0501 & .0758 & .1079 & .1463 & 1917. & .2387 & 2910 & .3490 & .4064 & 4698 & .5269 \\
\hline 2 & 5 & $w^{*}$ & .0503 & .0852 & .1285 & .1822 & 2413 & .3055 & .3755 & .4467 & .5179 & .5895 & .6562 \\
\hline 2 & 5 & $\hat{D}_{k, n}$ & .0490 & .0975 & 1490 & .2094 & 2717 & .3396 & .4047 & .4720 & .5392 & .6057 & .6666 \\
\hline & & & & & & & & & & & & & \\
\hline 5 & 2 & $J_{k, n}$ & .0500 & .0694 & .0930 & .1189 & .1437 & .1750 & 2046 & 2418 & 2777 & 3148 & 3505 \\
\hline 5 & 2 & $N_{k, n}$ & .0484 & .0673. & .0861 & . 1081 & .1346 & 1562 & 1873 & .2140 & 2464 & 2770 & .3086 \\
\hline 5 & 2 & $s_{k, n}$ & .0500 & .0660 & .0868 & .1080 & .1309 & .1551 & .1792 & .2098 & .2334 & .2666 & 2930 \\
\hline 5 & 2 & $A_{k, n}$ & .0506 & .0681 & .0881 & .1117 & .1377 & .1647 & .1940 & .2248 & .2543 & .2919 & .3299 \\
\hline 5 & 2 & $W^{*}$ & .0518 & .0703 & .0916 & .1168 & .1420 & .1698 & 2001 & .2317 & .2616 & .2973 & .3291 \\
\hline 5 & 2 & $\hat{D}_{k, n}$ & .0494 & .0706 & .0927 & .1194 & .1430 & .1707 & .1993 & .2289 & .2568 & .2879 & .3205 \\
\hline 4 & 5 & $J_{k, n}$ & .0504 & .0866 & .1341 & .1892 & .2538 & .3229 & .3980 & .4762 & .5509 & .6254 & 6939 \\
\hline 4 & 5 & $N_{k, n}$ & .0500 & .0798 & .1178 & .1592 & 2079 & .2634 & .3205 & .3818 & .4444 & .5066 & .5674 \\
\hline 4 & 5 & $s_{k, n}$ & .0502 & .0765 & .1133 & .1535 & .1998 & .2477 & .3010 & .3592 & .4154 & .4728 & .5274 \\
\hline 4 & 5 & $A_{k, n}$ & .0504 & .0771 & .1136 & .1580 & .2064 & .2615 & .3224 & .3836 & .4482 & .5120 & .5748 \\
\hline 4 & 5 & $W^{*}$ & .0488 & .0842 & .1292 & .1816 & .2423 & .3062 & .3730 & .4428 & .5106 & .5796 & .6430 \\
\hline 4 & 5 & $\hat{D}_{k, n}$ & .0519 & .0854 & .1267 & .1750 & .2262 & .2790 & .3378 & .3929 & .4582 & .5134 & .5716 \\
\hline 5 & 4 & $J_{k, n}$ & .0483 & .0784 & 1190 & .1635 & 2159 & .2750 & .3349 & .4004 & .4659 & .5369 & .5949 \\
\hline 5 & 4 & $N_{k, n}$ & .0492 & .0742 & .1045 & .1407 & .1805 & .2258 & .2718 & .3214 & .3775 & .4298 & .4812 \\
\hline 5 & 4 & $s_{k, n}$ & .0488 & .0744 & .1029 & .1364 & .1750 & .2172 & .2608 & .3058 & .3555 & 4065 & .4530 \\
\hline 5 & 4 & $A_{k, n}$ & .0488 & .0750 & .1060 & .1434 & .1867 & .2333 & .2829 & .3386 & .3955 & .4510 & .5105 \\
\hline 5 & 4 & $w^{*}$ & .0495 & .0804 & .1136 & .1569 & .2048 & .2557 & .3103 & .3695 & .4285 & .4858 & .5485 \\
\hline 5 & 4 & $\hat{\mathrm{D}}_{k, n}$ & .0518 & .0817 & .1144 & .1515 & .1936 & .2367 & .2795 & .3261 & .3782 & .4227 & .4710 \\
\hline
\end{tabular}

The comparison results in an exponential population are similar to those in a normal population. When the imperfect ranking scenarios are fraction of random ranking and fraction of inverse ranking (Tables 7,8 ) the proposed test is uniformly better than the other ones, but for fraction of neighbor ranking scenario (Table 9) the test based on $J_{k, n}$ generally has better powers in comparison with its competitor (except for $(k, n)=(2,5)$ where $\hat{D}_{k, n}$ is the best). 

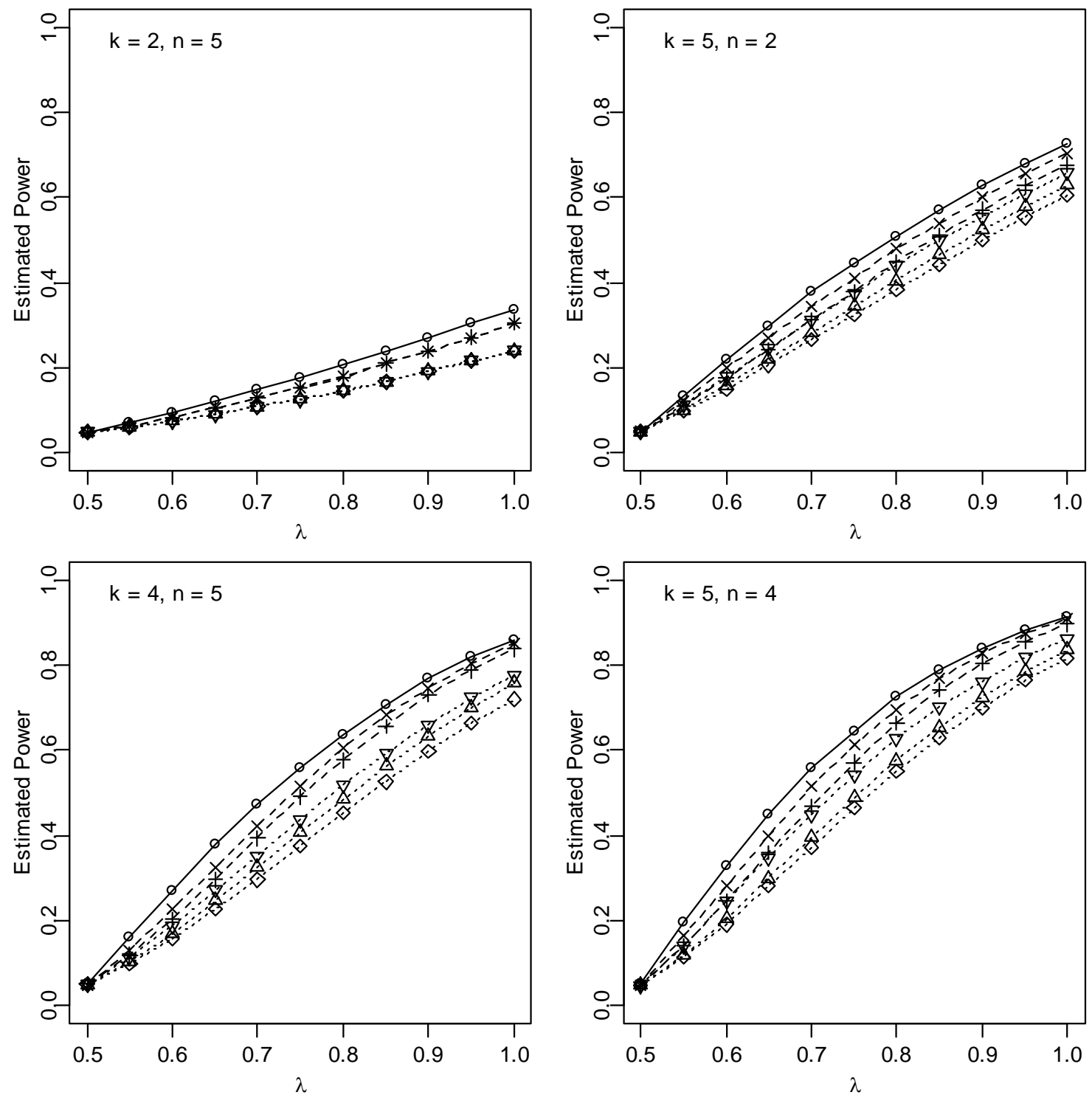

Figure 5. Visualized estimated powers of tests based on $J_{k, n}$ (represented by + ), $N_{k, n}$ (represented by $\Delta), S_{k, n}($ represented by $\nabla), A_{k, n}$ (represented by $\left.\diamond\right), W^{*}$ (represented by $\times), D_{k, n}$ (represented by $\bigcirc$ ) in Table 7 under the assumption of exponentiality and fraction of random rankings model with fraction $\lambda$ at significance level $\alpha=0.05$. 

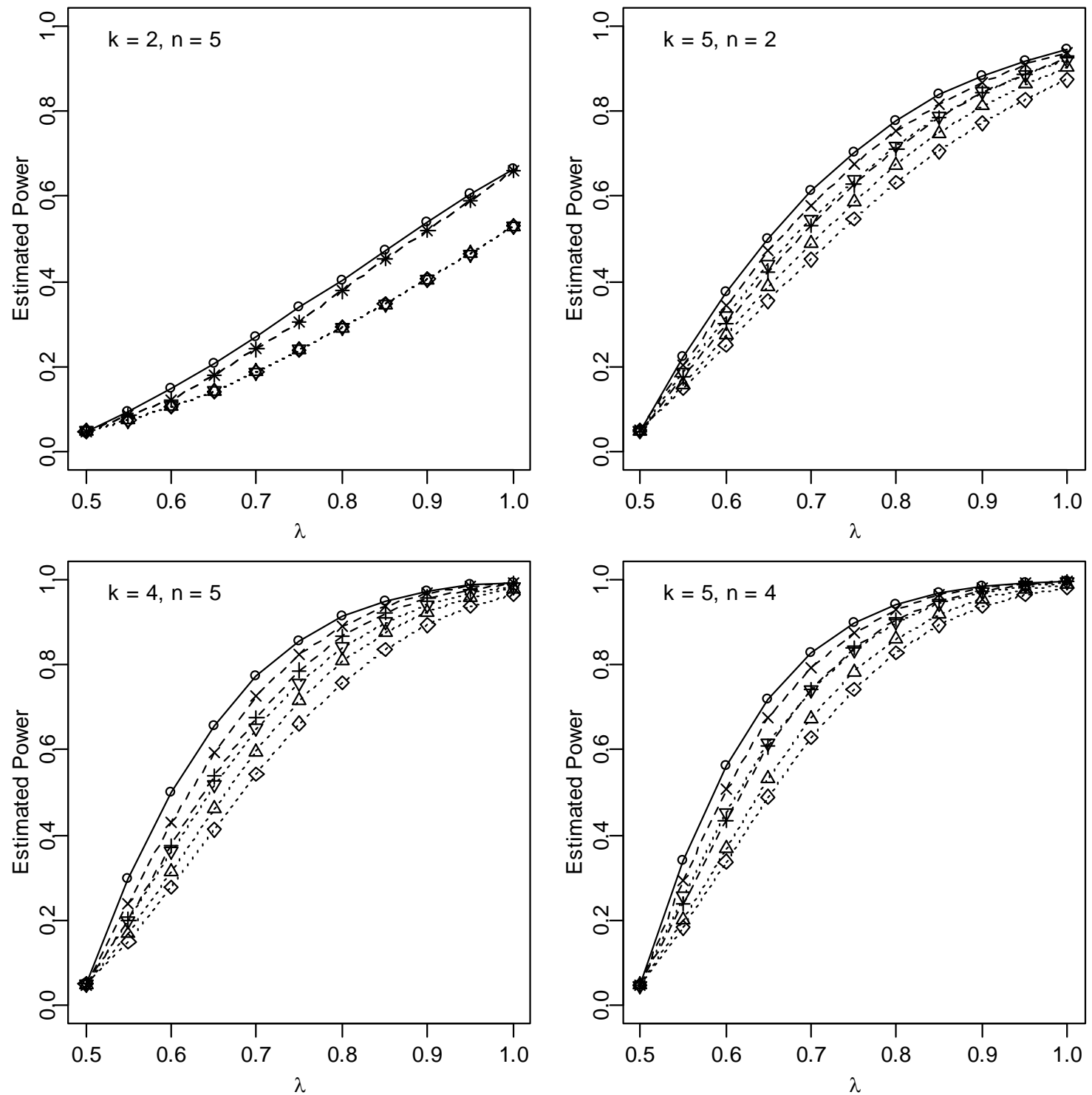

Figure 6. Visualized estimated powers of tests based on $J_{k, n}($ represented by +$), N_{k, n}$ (represented by $\Delta), S_{k, n}($ represented by $\nabla), A_{k, n}$ (represented by $\left.\diamond\right), W^{*}$ (represented by $\times), D_{k, n}$ (represented by $\bigcirc$ ) in Table 8 under the assumption of exponentiality and fraction of inverse rankings model with fraction $\lambda$ at significance level $\alpha=0.05$. 

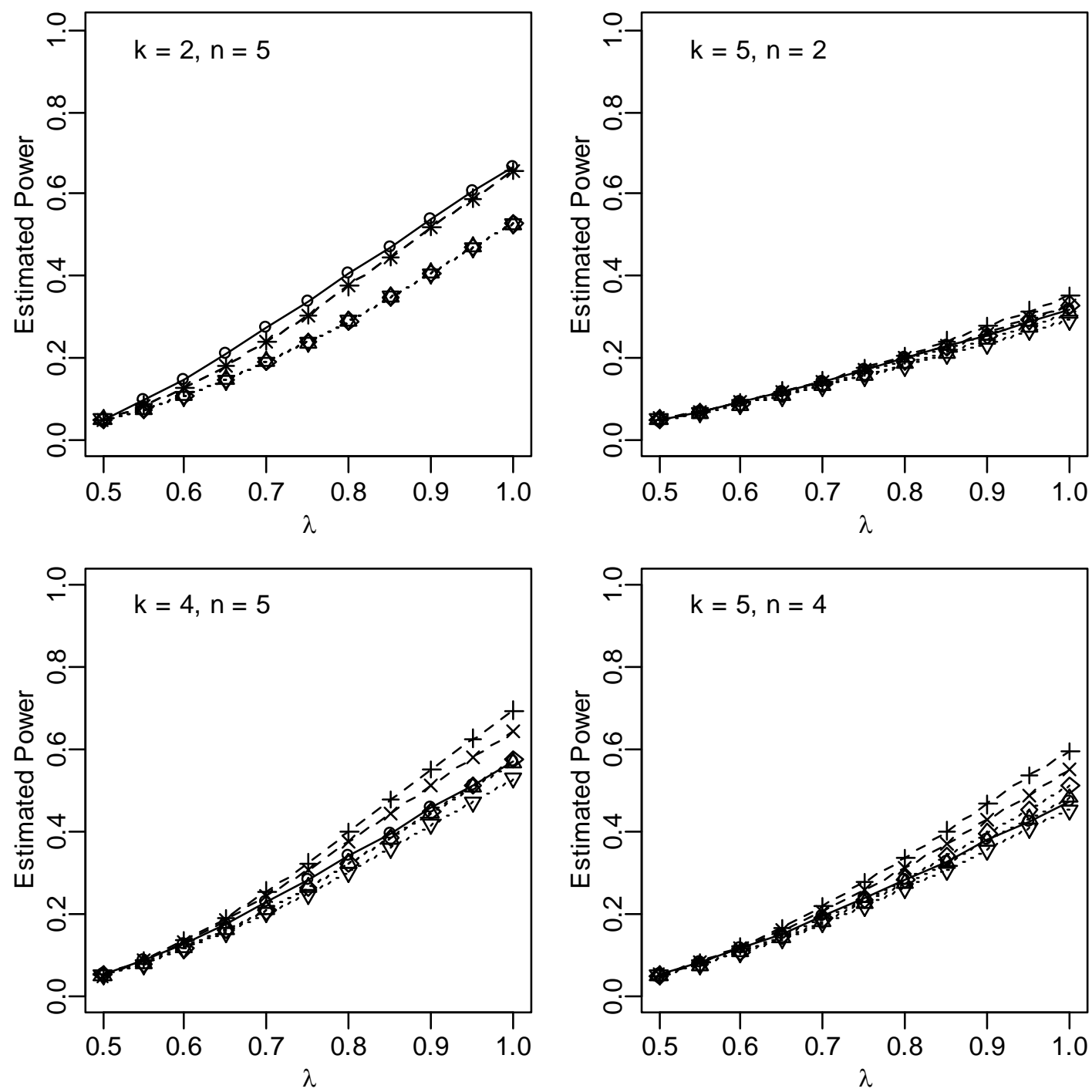

Figure 7. Visualized estimated powers of tests based on $J_{k, n}($ represented by +$), N_{k, n}$ (represented by $\Delta), S_{k, n}$ (represented by $\left.\nabla\right), A_{k, n}($ represented by $\diamond), W^{*}$ (represented by $\times), D_{k, n}$ (represented by $\bigcirc$ ) in Table 9 under the assumption of exponentiality and fraction of neighbors model with fraction $\lambda$ at significance level $\alpha=0.05$. 


\section{Conclusion}

In this paper, we propose a parametric test of perfect ranking in balanced ranked set samples. We show that our test is location free and it will be scale free if we use an equivariant estimator for the scale parameter. Then we compare our test with its leading competitors in two cases of normality and exponentiality. Our simulation results show that the proposed test is quite competitive, especially when ranking errors in the data can be severe (fraction of random ranking and fraction of inverse ranking), in which cases the proposed test is uniformly the best. Therefore, since most statistical methods are robust against mild ranking errors, we recommend using our proposed test in practice when there is an assumption about the population distribution. The advantage of our proposed test is higher power when it can be assumed that the unknown distribution belongs to a certain known location-scale family. Therefore our proposed test should be used with care if there are doubts about the correctness of the above assumption.

Acknowledgements: Some part of this work had been done while the first author was spending his sabbatical leave at University of Bern, Bern, Switzerland. The authors are thankful to an anonymous referee for comments which led to an improvement to the earlier version of this paper.

\section{Appendix}

\section{Proof of Theorem 1:}

Let $X_{(i), k}$ be the $i^{\text {th }}$ order statistics from a sample of size $k$, and then its pdf and cdf can be written as

$$
\begin{aligned}
& f_{i, k}(x)=i\left(\begin{array}{l}
k \\
i
\end{array}\right)(F(x))^{i-1}(1-F(x))^{k-i} f(x), \\
& F_{i, k}(x)=\sum_{j=i}^{k}\left(\begin{array}{l}
k \\
j
\end{array}\right)(F(x))^{j}(1-F(x))^{k-j} .
\end{aligned}
$$

Let $Y=I\left(X_{(j), k}>X_{(l), k}\right)$, then we have:

$$
E\left(\left(X_{(j) \cdot k}-X_{(l), k}\right) I\left(X_{(j), k}>X_{(l), k}\right)\right)=E\left(X_{(j), k} Y\right)-E\left(X_{(l), k} Y\right),
$$

but

$$
\begin{aligned}
& E\left(X_{(j), k} Y\right)=E\left(X_{(j), k} Y \mid Y=1\right) P(Y=1)+E\left(X_{(j), k} Y \mid Y=0\right) P(Y=0) \\
& =\frac{\int_{0}^{+\infty} x_{(j)} P\left(X_{(l), k}<x_{(j)}\right) f_{j, k}\left(x_{(j)}\right) d x_{(j)}}{P(Y=1)} \times P(Y=1)=\int_{0}^{+\infty} x_{(j)} P\left(X_{(l), k}<x_{(j)}\right) f_{j, k}\left(x_{(j)}\right) d x_{(j)}
\end{aligned}
$$

Let $t=x_{(j)}$, then we can rewrite the above equation as 


$$
\begin{aligned}
& \left.\left.=\int_{0}^{+\infty} t\left(\begin{array}{l}
k \\
k_{1}=l \\
k \\
k_{1}
\end{array}\right) F^{k_{1}}(t)(1-F(t))^{k-k_{1}}\right)\left(\begin{array}{l}
k \\
j
\end{array}\right) F^{j-1}(t)(1-F(t))^{k-j}\right) f(t) d t \\
& =\sum_{k_{1}=l}^{k} j\left(\begin{array}{l}
k \\
k_{1}
\end{array}\right)\left(\begin{array}{l}
k \\
j
\end{array}\right) \int_{0}^{+\infty} t(F(t))^{k_{1}+j-1}(1-F(t))^{2 k-\left(k_{1}+j\right)} f(t) d t \\
& =\sum_{k_{1}=l}^{k} \frac{j\left(\begin{array}{l}
k \\
k_{1}
\end{array}\right)\left(\begin{array}{l}
k \\
j
\end{array}\right)}{\left(k_{1}+j\right)\left(\begin{array}{l}
2 k \\
k_{1}+j
\end{array}\right)} \int_{0}^{+\infty} t\left(k_{1}+j\right)\left(\begin{array}{l}
2 k \\
k_{1}+j
\end{array}\right)(F(t))^{k_{1}+j-1}(1-F(t))^{2 k-\left(k_{1}+j\right)} f(t) d t \\
& =\sum_{k_{1}=l}^{k} \frac{j\left(\begin{array}{l}
k \\
k_{1}
\end{array}\right)\left(\begin{array}{l}
k \\
j
\end{array}\right)}{\left(k_{1}+j\right)\left(\begin{array}{l}
2 k \\
k_{1}+j
\end{array}\right)} \int_{0}^{+\infty} t f_{k_{1}+j, 2 k}(t) d t=\sum_{k_{1}=l}^{k} \frac{j\left(\begin{array}{l}
k \\
k_{1}
\end{array}\right)\left(\begin{array}{l}
k \\
j
\end{array}\right)}{\left.k_{1}+j\right)\left(\begin{array}{l}
2 k \\
k_{1}+j
\end{array}\right)} E\left(X_{\left(k_{1}+j\right), 2 k}\right) .
\end{aligned}
$$

Similarly, it can be shown that

$$
E\left(X_{(l), k} Y\right)=E_{l, k}-\sum_{k_{1}=j}^{k} \frac{l\left(\begin{array}{l}
k \\
k_{1}
\end{array}\right)\left(\begin{array}{l}
k \\
l
\end{array}\right)}{\left(k_{1}+l\right)\left(\begin{array}{l}
2 k \\
k_{1}+l
\end{array}\right)} E_{k_{1}+l, 2 k} .
$$

The Theorem is proved by substituting (11) and (12) in (10).

\section{Critical values of the test statistic under a $\boldsymbol{t}$ distribution:}

Tables 10-14 show the critical values of $\hat{D}_{k, n}$ at significance level $\alpha=.05$ based on 100,000 repetitions, assuming that the population distribution is a $t$-distribution with $\mathrm{df}=2,3,4,5,10$ and for different values of $k$ and $n$.

Table 10. Critical values of $\hat{D}_{k, n}$ at significance level $\alpha=.05$, assuming that the population distribution is $t(2)$

\begin{tabular}{|r|r|r|r|r|r|r|r|r|r|}
\hline$n$ & 2 & 3 & 4 & 5 & 6 & 7 & 8 & 9 & 10 \\
\hline 1 & 10.19 & 11.05 & 23.78 & 30.21 & 38.70 & 49.64 & 58.19 & 69.55 & 82.50 \\
\hline 2 & 30.13 & 43.29 & 63.23 & 90.23 & 117.93 & 154.63 & 185.64 & 224.10 & 262.97 \\
\hline 3 & 42.27 & 72.93 & 119.61 & 167.53 & 228.25 & 294.22 & 370.94 & 446.57 & 535.71 \\
\hline 4 & 56.46 & 106.93 & 180.71 & 265.57 & 365.43 & 471.48 & 599.33 & 736.01 & 876.25 \\
\hline 5 & 72.31 & 144.82 & 248.85 & 381.37 & 524.67 & 694.28 & 869.88 & 1062.60 & 1281.33 \\
\hline
\end{tabular}


Table 11. Critical values of $\hat{D}_{k, n}$ at significance level $\alpha=.05$, assuming that the population distribution is $t(3)$

\begin{tabular}{|r|r|r|r|r|r|r|r|r|r|}
\hline \multicolumn{1}{|c|}{$n^{k}$} & 2 & 3 & 4 & 5 & 6 & 7 & 8 & 9 & 10 \\
\hline 1 & 11.57 & 12.75 & 26.95 & 35.02 & 45.74 & 57.40 & 70.93 & 83.72 & 97.35 \\
\hline 2 & 34.53 & 49.87 & 73.62 & 107.81 & 143.45 & 183.57 & 234.62 & 286.32 & 336.32 \\
\hline 3 & 48.63 & 86.48 & 142.24 & 205.03 & 279.23 & 370.60 & 473.84 & 570.62 & 680.10 \\
\hline 4 & 64.88 & 128.44 & 222.75 & 326.32 & 457.98 & 612.30 & 782.82 & 971.59 & 1160.92 \\
\hline 5 & 83.01 & 176.72 & 312.85 & 477.09 & 670.07 & 889.83 & 1142.16 & 1438.50 & 1703.20 \\
\hline
\end{tabular}

Table 12. Critical values of $\hat{D}_{k, n}$ at significance level $\alpha=.05$, assuming that the population distribution is $t(4)$

\begin{tabular}{|c|c|c|c|c|c|c|c|c|c|}
\hline$k$ & 2 & 3 & 4 & 5 & 6 & 7 & 8 & 9 & 10 \\
\hline 1 & 12.26 & 13.55 & 29.37 & 37.83 & 49.24 & 60.34 & 75.00 & 91.58 & 108.09 \\
\hline 2 & 36.48 & 54.44 & 81.20 & 117.31 & 156.86 & 208.07 & 257.59 & 316.57 & 367.01 \\
\hline 3 & 51.20 & 92.84 & 151.04 & 224.08 & 314.15 & 416.68 & 518.15 & 648.48 & 768.22 \\
\hline 4 & 68.33 & 139.99 & 240.50 & 357.11 & 508.00 & 686.15 & 877.56 & 1079.93 & 1290.92 \\
\hline 5 & 87.78 & 193.67 & 344.35 & 529.53 & 743.45 & 1003.05 & 1295.63 & 1610.25 & 1962.49 \\
\hline
\end{tabular}

Table 13. Critical values of $\hat{D}_{k, n}$ at significance level $\alpha=.05$, assuming that the population distribution is $t(5)$

\begin{tabular}{|c|c|c|c|c|c|c|c|c|c|}
\hline & 2 & 3 & 4 & 5 & 6 & 7 & 8 & 9 & 10 \\
\hline 1 & 12.67 & 14.83 & 30.55 & 38.86 & 49.98 & 63.45 & 79.90 & 95.24 & 114.23 \\
\hline 2 & 37.52 & 56.57 & 85.02 & 122.89 & 164.30 & 216.97 & 270.91 & 330.03 & 391.87 \\
\hline 3 & 53.44 & 98.47 & 162.96 & 237.22 & 332.91 & 443.71 & 553.28 & 677.76 & 832.38 \\
\hline 4 & 71.95 & 148.83 & 253.43 & 383.97 & 545.74 & 721.43 & 929.88 & 1143.61 & 1372.14 \\
\hline 5 & 91.08 & 203.26 & 362.33 & 562.92 & 788.65 & 1067.73 & 1358.85 & 1735.91 & 2090.60 \\
\hline
\end{tabular}

Table 14. Critical values of $\hat{D}_{k, n}$ at significance level $\alpha=.05$, assuming that the population distribution is $t(10)$

\begin{tabular}{|r|r|r|r|r|r|r|r|r|r|}
\hline \multicolumn{1}{|r|}{$n$} & 2 & \multicolumn{1}{c|}{3} & 4 & 5 & \multicolumn{1}{c|}{6} & \multicolumn{1}{c|}{7} & 8 & 9 & \multicolumn{1}{c|}{10} \\
\hline 1 & 13.48 & 15.47 & 31.95 & 41.56 & 55.33 & 70.41 & 86.71 & 104.19 & 124.06 \\
\hline 2 & 40.55 & 61.49 & 90.19 & 133.22 & 181.65 & 239.57 & 296.73 & 371.25 & 444.59 \\
\hline 3 & 56.94 & 106.56 & 176.53 & 259.84 & 368.42 & 483.16 & 614.48 & 767.99 & 925.30 \\
\hline 4 & 76.70 & 160.34 & 278.24 & 428.16 & 602.10 & 804.07 & 1046.18 & 1284.80 & 1559.03 \\
\hline 5 & 99.27 & 221.98 & 403.47 & 623.95 & 889.90 & 1194.85 & 1546.27 & 1927.02 & 2353.19 \\
\hline
\end{tabular}




\section{References:}

Arnold, B.C., Balakrishnan, N. and Nagaraja, H.N., 1992. A First Course in Order Statistics. Wiley, New York.

Balakrishnan, N. and Li, T., 2005. BLUEs of Parameters of Generalized Geometric Distribution Using Ordered Ranked Set Sampling. Communications in Statistics Simulation and Computation 34, 253-266.

Bhoj, D. S., Ahsanullah, M., 1996. Estimation of parameters of the generalized geometric distribution using ranked set sampling. Biometrics 52, 685-694.

Frey, J., Ozturk, O., and Deshpande, J.V., 2007. Nonparametric tests for perfect judgment rankings. Journal of the American Statistical Association 102, 708-717.

Li, T. and Balakrishnan, N, 2008. Some simple nonparametric methods to test for perfect ranking in ranked set sampling. Journal of Statistical Planning and Inference 138, 13251338.

McIntyre, G. A., 1952. A method for unbiased selective sampling using ranked set sampling. Austral. J. Agricultural Res. 3, 385-390.

Perron, F., and Sinha, B.K., 2004. Estimation of variance based on ranked set samples. Journal of Statistical Planning and Inference 120, 21-28.

Stokes, S.L., 1980. Estimation of variance using judgement ordered ranked set samples. Biometrics 36, 35-42.

Sengupta, S. and Mukhuti, S., 2006. Unbiased variance estimation in a simple exponential population using ranked set samples. Journal of Statistical Planning and Inference 136, 1526-1553.

Takahasi, K. and Wakimoto, K., 1968. On unbiased estimates of the population mean based on the sample stratified by means of ordering, Ann. Inst. Statist. Math. 21, 249255.

Vock, M. and Balakrishnan, N., 2011. A Jonckheere-Terpstra-type test for perfect ranking in balanced ranked set sampling, Journal of Statistical Planning and Inference $141,624-630$.

Vock, M. and Balakrishnan, N., 2012. A connection between two nonparametric tests for perfect ranking in balanced ranked set sampling, To appear in Communication in Statistics-Theory and Methods

Wolfe, D. A., 2004. Ranked set sampling; an approach to more efficient data collection. Statistical Science 19, 636-643. 